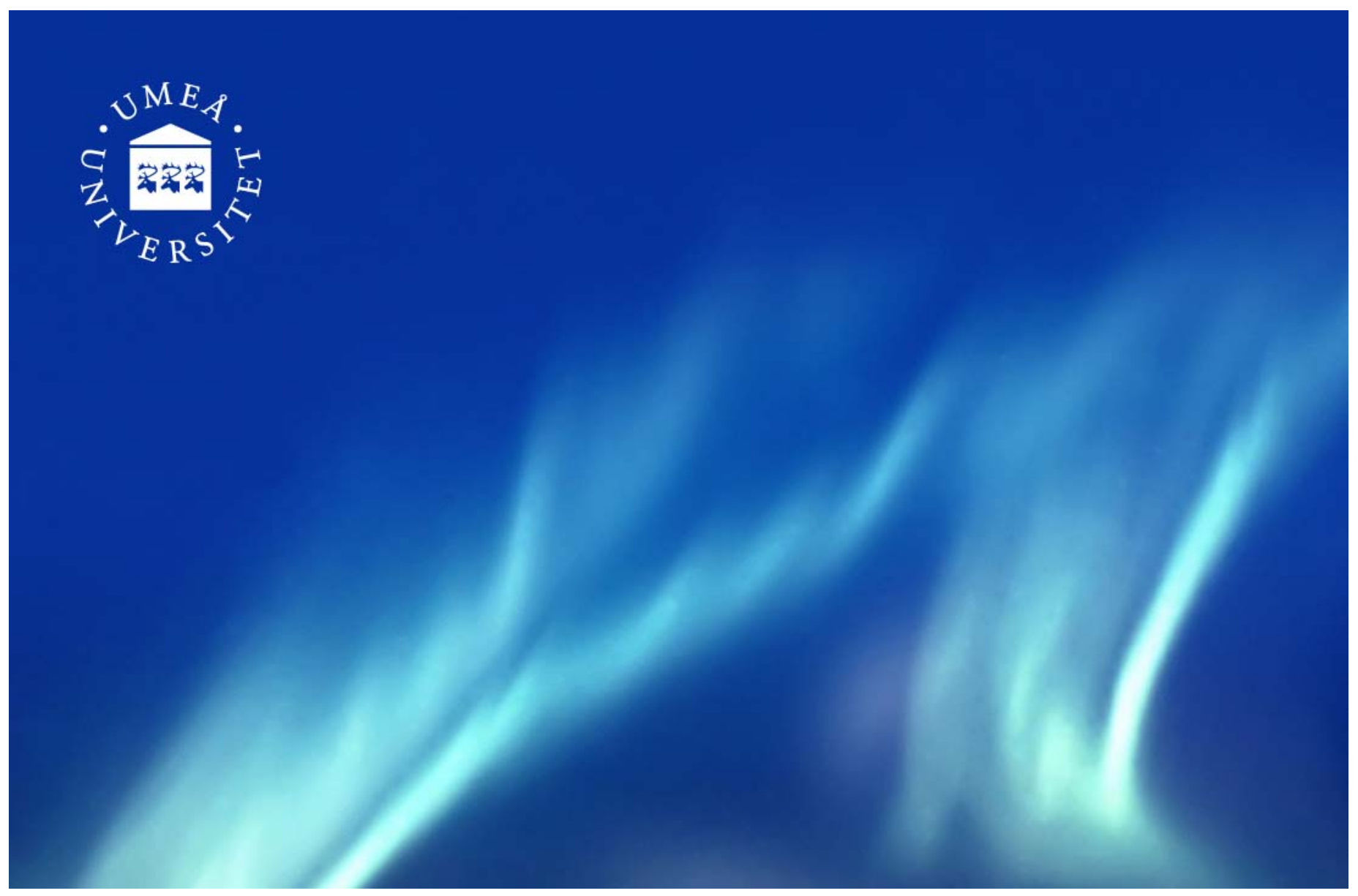

DiVA - Digitala Vetenskapliga Arkivet http://umu.diva-portal.org

This is an author produced version of a paper published in Space Science Reviews

This paper has been peer-reviewed but does not include the final publisher proof-corrections or journal pagination.

Citation for the published paper:

Ingrid Mann, Asta Pellinen-Wannberg, Edmond Murad, Olga Popova, Nicole Meyer-Vernet, Marlene Rosenberg, Tadashi Mukai, Andrzej Czechowski, Sonoyo Mukai, Jana Safrankova, Zdenek Nemecek

Dusty plasma effects in near earth space and interplanetary medium

Space Science Reviews, 2011, Vol. 161, Issue 1-4: 1-47

10.1007/s11214-011-9762-3

Access to the published version may require subscription. Published with permission from:

Springer Science+Business Media B.V. 


\title{
Dusty plasma effects in near Earth space and interplanetary medium
}

\author{
Ingrid Mann · Asta Pellinen-Wannberg · Edmond \\ Murad · Olga Popova · Nicole Meyer-Vernet · \\ Marlene Rosenberg · Tadashi Mukai · Andrzej \\ Czechowski · Sonoyo Mukai · Jana Safrankova · \\ Zdenek Nemecek
}

Received: date / Accepted: date

\begin{abstract}
We review dust and meteoroid fluxes and their dusty plasma effects in the interplanetary medium near Earth orbit and in the Earth's ionosphere. Aside from in-situ measurements from sounding rockets and spacecraft, experimental data cover radar and optical observations of meteors. Dust plasma interactions in the interplanetary medium are observed by the detection of charged dust particles, by the detection of dust that is accelerated in the solar wind and by the detection of ions and neutrals that are released from the dust. These interactions are not well understood and lack quantitative description. There is still a huge discrepancy in the estimates of meteoroid mass deposition into the atmosphere. The radar
\end{abstract}

Ingrid Mann

Belgian Institute for Space Aeronomy

Tel.: + 3223730406

Fax: + 3223748423

E-mail: ingrid.mann@aeronomie.be

3 Avenue Circulaire, 1180 Brussels, Belgium

Asta Pellinen-Wannberg

Umeå University and Swedish Institute of Space Physics, Kiruna, Sweden

Edmond Murad

Newton, USA

Olga Popova

Institute for Dynamics of Geospheres, Russian Academy of Science, Moscow, Russia

Nicole Meyer-Vernet

Observatoire de Paris, Meudon, France

Marlene Rosenberg

Dept. of Electrical \& Computer Engineering, Univ. of California, San Diego, USA

Tadashi Mukai

Kobe University, Japan

Andrzej Czechowski

Polish Space Research Institute, Warsaw, Poland

Sonoyo Mukai

Kinki University, Japan

Jana Safrankova and Zdenek Nemecek

Charles University, Prague, Czech Republic 
meteor observations are of particular interest for determining this number. Dust measurements from spacecraft require a better understanding of the dust impact ionization process, as well as of the dust charging processes. The latter are also important for further studying nano dust trajectories in the solar wind. Moreover understanding of the complex dependencies that cause the variation of nano dust fluxes is still a challenge.

Keywords cosmic dust $\cdot$ meteors $\cdot$ aerosols $\cdot$ interplanetary medium $\cdot$ solar wind · ionosphere $\cdot$ Earth atmosphere $\cdot$ dusty plasma

\section{Introduction}

Plasmas containing charged dust particles of sizes $<\mathrm{mm}$ are loosely referred to as dusty plasmas. The field of dusty plasma studies has grown rapidly over the last 15 years, driven by applications to astrophysical, space and laboratory plasmas. Dust is ubiquitous in the cosmos, occurring in interstellar, circumstellar, interplanetary, circumplanetary, and cometary environments. Dust also plays an important role in atmospheric processes. It is studied in laboratory plasmas, including plasmas used for materials processing, fusion plasmas, and dedicated dusty plasma experiments. Many processes in astrophysics rely on dust-plasma interactions and they often play a notable role, as, for instance, in the evolution of the interstellar medium.

Although the parameters of dusty plasmas cover an extremely wide range, certain fundamental concepts are common and may have application to many of these environments. For example, dust in a plasma is generally charged due to various mechanisms such as the collection of plasma electrons and ions. As another example, there are various forces that can act on the charged dust component, which are not usually considered for the much lighter ions and electrons such as gravitational forces, drag due to relative motion between dust and plasma or gas, and radiation pressure forces. And as a further example, charged dust can affect the plasma collective behavior, modifying waves and instabilities and leading to new very low frequency waves and instabilities associated with the dust.

Most of the dust-plasma interactions are at present not directly observed in space and the progress in the theory of dusty plasmas stands in contrast to the small amount of available data on the microphysics of dust-plasma interactions. The recent discussions of neutral solar wind, of inner source pick-up ions and of interplanetary field enhancements (see Section 4) suggest dust plasma interactions as a cause of these phenomena, but fail to provide the complete explanation.

Though the dust particles in the interplanetary medium and in near Earth space are immersed in plasmas with quite different parameters, what they have in common is that the space measurements provide direct access to them. The dust and larger meteoroids that cross the Earth orbit generate the dust in the ionosphere. The dust in the ionosphere is detected from sounding rockets and observed in meteors ${ }^{1}$

In Section 2 we describe the dust and plasma environment in the interplanetary medium and near Earth space. We discuss the dust flux onto the Earth and give an overview of

\footnotetext{
1 According to the convention of the International Astronomical Union a meteor is in particular, the light phenomenon, which results from the entry into (usually the Earth's) atmosphere of a solid particle from space; and more generally any physical object or phenomenon associated with such an event. Meteoroids are bodies (usually in the interplanetary medium) that are considerably smaller than an asteroid and considerably larger than an atom or molecule. Meteorites are those parts of meteoroids that reach the surface of the Earth without being completely vaporized and "meteoric" is the adjectival form pertaining to meteors and meteoroids. Interplanetary dust particles are micrometeoroids typically of sizes of $100 \mu \mathrm{m}$ and smaller. (cf. Mann 2009).
} 
the different dust - plasma interactions. In Section 3 we describe dust measurements in the ionosphere based on observations of meteors, the light and ionization that is caused by meteoroids entering the Earth' $s$ ionosphere. We further review the detection of dust by rocket-borne experiments and Radar and we discuss the possible formation of dust-plasma instabilities. In Section 4 we discuss the dust in the interplanetary medium near Earth orbit and the observation of dust generated neutrals and ions in the solar wind as well as the acceleration of nano dust in the solar wind. The Section 5 is devoted to dust charging and laboratory experiments related to dust in space. The physical principles of dust instruments are discussed in and Section 6 . Section 7 is a summary. A summarizing paragraph precedes each section. Except otherwise stated, SI units are used throughout.

\section{The dust and plasma environments}

The interplanetary medium includes a highly ionized solar wind and slowly flowing neutral gas. It is characterized by high plasma temperatures and small dust densities. There is a continuous distribution of objects from $\mathrm{nm}$ to the $\mathrm{km}$ sizes. The ionosphere has a low degree of ionization and a high gas density. Estimates of the amount of meteoroid material that enters from the interplanetary medium vary by orders of magnitude, but agree that the peak of this mass flux is approximately in the mass range of objects observed as radar meteors. The meteoroid material that remains in the atmosphere forms observed heavy ion layers. A fraction of this meteoroid material condenses into meteoric smoke particles. These are of nanometric size and were recently observed for the first time.

We describe the interplanetary medium in section (2.1) and the upper Earth' s atmosphere in section (2.2). Section (2.3) discusses the different estimates of the amount of meteoroid material that enters the Earth atmosphere and the final section (2.4) the possibly forming dust - plasma interactions.

\subsection{Interplanetary medium}

The solar wind plasma is the major component of the interplanetary medium. Following Meyer-Vernet (2007) we summarize some of its major characteristics. The solar wind has speeds on the order of $300-800 \mathrm{~km} / \mathrm{s}$ and an average plasma density of $5 \times 10^{6} \mathrm{~m}^{-3}$ near $1 \mathrm{AU}$ (e.g. Table 2.4_2). In the fast wind, which comes from the solar polar regions, the density is $\sim 2-3 \times 10^{6} \mathrm{~m}^{-3}$ and the speed $\sim 700-800 \mathrm{~km} / \mathrm{s}$. The slow wind has the density $\sim 7 \times 10^{6} \mathrm{~m}^{-3}$ and speed $\sim 300-500 \mathrm{~km} / \mathrm{s}$. During ejections of coronal material, densities can reach $\sim 10^{8} \mathrm{~m}^{-3}$. Near the Sun, in the F-corona, the slow wind can have speed $\sim 100$ $\mathrm{km} / \mathrm{s}$ and density $\sim 10^{11} \mathrm{~m}^{-3}$ at around 4 solar radii, while coronal mass ejections are associated with denser plasmas that are faster than typical solar wind speed (see St Cyr et al. 2000). The solar wind drags the solar magnetic field, so that the magnetic field in the slow solar wind has a radial component of the order of $3 \mathrm{nT}$ at $1 \mathrm{AU}$, whereas the solar rotation produces a spiral shape with an azimuthal magnetic field of roughly $3 \mathrm{nT}$ at $1 \mathrm{AU}$ in the ecliptic.

The vast majority of ions in the interplanetary medium are protons and $\alpha$-particles, followed by highly ionized heavy ions. The measurements of particle fluences from roughly 0.3 $\mathrm{keV} /$ nucleon to $300 \mathrm{MeV} /$ nucleon in the interplanetary medium were recently summarized by Mewaldt et al. (2007) and this gives the following picture: The solar wind contributes the majority of particles in the energy range up to $\sim 8 \mathrm{keV} /$ nucleon. It varies on a typical 
scale of a factor of $\sim 2$ in velocity, flux, and composition and on time scales that range from hours to days to years. Intermediate range of energies between $\sim 10 \mathrm{keV} /$ nucleon and $\sim$ $5 \mathrm{MeV} /$ nucleon is covered by different types of particles and the fluences are more timevariable and difficult to predict. Solar energetic particles make the largest contribution at energies $\sim 5$ to $\sim 50 \mathrm{MeV} /$ nucleon, and galactic cosmic rays at even larger energies. The galactic and anomalous cosmic rays are rather stable in composition, while their energy spectra vary.

Asteroids and comets generate the majority of dust and meteoroids in the interplanetary medium and smaller dust particles subsequently form by collisional fragmentation. The interstellar dust provides a further component (see e.g. Mann et al. 2004). The dust velocities relative to their parent bodies are typically comparatively small and dust particles initially move with the velocities of the (Keplerian) orbits of the parent-bodies. The lifetime of dust roughly larger than $\mu \mathrm{m}$ is limited by mutual collisions and by the Poynting-Robertson effect (see 4.3) that results from radiation pressure force. Smaller particles are in majority ejected from the inner solar system.

\subsection{The upper Earth's atmosphere}

The Earth is shielded from the solar wind by its magnetic field that gives rise to the magnetospheres which ranges roughly 10 to 12 earth radii $(70000 \mathrm{~km})$ from the center of the Earth in the sunward direction and more than 200 Earth radii in the direction away from the Sun. While it shields the Earth's atmosphere from the solar wind, meteoroids and dust can freely enter. Meteors are observed from about $70 \mathrm{~km}$ to about $400 \mathrm{~km}$ (see Section 3). This altitude range covers most of what is typically referred to as "ionosphere" and comprises the D, E and F layers. Transitions between those layers are variable. The layers were initially distinguished by their distinct reflections of radio waves and their composition is influenced by absorption of solar radiation. The $\mathrm{D}$ region extends below $90 \mathrm{~km}$, the $\mathrm{E}$ region between 90 and $150 \mathrm{~km}$ and the F region between 150 and $500 \mathrm{~km}$ (following Kelley 1989).

In terms of temperature the region of interest lies mainly within the thermosphere. This is the region above the mesopause, which is defined as the altitude of minimum temperature. Temperatures, as well as the location of the mesopause are highly variable. LIDAR observations at two mid-latitude sites during night time, for instance, give mesopause altitudes of $101 \mathrm{~km}$ in winter and at $85 \mathrm{~km}$ during summer, while in spring and autumn the altitude oscillates between these states (Plane et al. 1999). The plasma is generally cold, with temperatures ranging from about a few hundred $\mathrm{K}$ at low altitudes (or even colder in the summer mesopause) to about $2000 \mathrm{~K}$ at higher altitudes in daytime, and about $1000 \mathrm{~K}$ in nighttime. Electron temperature is typically larger than ion and neutral temperatures.

The altitude profiles of the dominant neutral atmospheric constituents $\mathrm{N}_{2}, \mathrm{O}_{2}, \mathrm{O}$ as well as the dominant ionized constituents $\mathrm{NO}^{+}, \mathrm{O}_{2}^{+}, \mathrm{O}^{+}$and their variations due to the solar cycle, time of day, latitude, season and geomagnetic activity is quite well known above about $80 \mathrm{~km}$. The densities are highly variable from day to night and from year to year, most notably with solar cycle. Fig. 1 shows the profiles of these neutral and ionized atmospheric constituents in solar maximum and minimum conditions at night. The plasmas are weakly ionized (see Table 2.4_2). Note, that the lower part of the region that we consider is also the region where ionized species are molecular, primarily $\mathrm{NO}^{+}$; at altitudes lower than 80 $\mathrm{km}$ water hydrates such as $\mathrm{H}^{+}\left(\mathrm{H}_{2} \mathrm{O}\right)_{n>2}$ assume increasing importance and replace other diatomic ions (Kull et al. 1997). 


\section{Density profiles in solar max and min conditions}

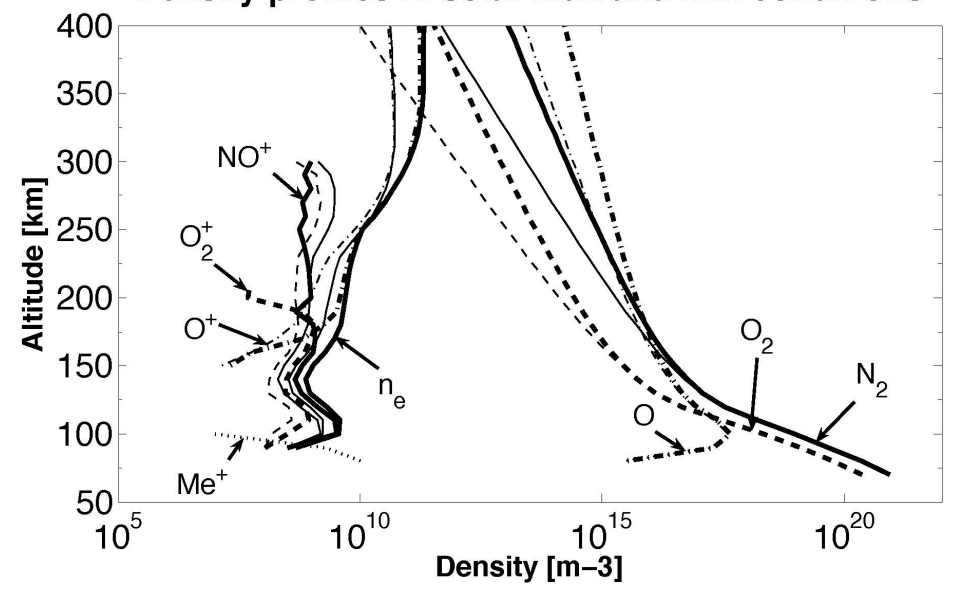

Fig. 1 Density profiles of the neutral atmosphere constituents $\mathrm{N}_{2}$ (solid), $\mathrm{O}_{2}$ (dashed) and $\mathrm{O}$ (dashed-dotted) under solar maximum (bold) and minimum conditions are located to the right in the density scale. Similarly the ionized components are shown as the total electron density profiles $\mathrm{n}_{e}$ (solid) in the middle and $\mathrm{NO}^{+}$ (solid), $\mathrm{O}_{2}^{+}$(dashed) and $\mathrm{O}^{+}$(dash-dotted) to the left. The dotted profile represents ions of meteoric origin, $\mathrm{Me}^{+}$. These profiles are generated from the IRI model (Bilitza and Reinisch 2008) for the ionosphere and MSIS model (Hedin 1991) for the atmosphere under solar maximum conditions in December 1990 and solar minimum conditions in December 1996 at location $60^{\circ} \mathrm{N}, 20^{\circ} \mathrm{E}$ at $22 \mathrm{UT}$. Densities are higher during daytime. The plasma (electron) density for the the same IRI model and day at 13.5 hours local time ranges from $7 \cdot 10^{9} \mathrm{~m}^{-3}$ at $90 \mathrm{~km}$ to $10^{12} \mathrm{~m}^{-3}$ at $400 \mathrm{~km}$. Neutral densities (from the MSIS model) range from about $5 \cdot 10^{18} \mathrm{~m}^{-3}$ at $90 \mathrm{~km}$ to $2 \cdot 10^{13} \mathrm{~m}^{-3}$ to $2 \cdot 10^{14} \mathrm{~m}^{-3}$ at $400 \mathrm{~km}$ (with the lower number for 1996). The meteoric ion profile is taken from Roble (1995).

\subsection{Dust flux in the solar wind and into Earth atmosphere}

The different estimates of meteoroid flux onto Earth vary by more than an order of magnitude, from 2 to $80 \cdot 10^{7} \mathrm{~kg} \mathrm{yr}^{-1}$ over the whole planet (note though that observed meteor fluxes vary significantly with location, diurnal and annually, see e.g. Szasz et al. 2004). They are based on studies of dust and meteoroids outside of the atmosphere (Hughes 1978; Grün et al. 1985; Love and Brownlee 1993; and others), on atmospheric studies, as well as on studies of the deposition of extraterrestrial matter in glaciers and sediments (see Kane and Gardner 1993; Taylor et al. 1998; Mathews et al. 2001; Cziczo et al. 2001; Gabrielli et al. 2004; and others). Aside from the differences between the different observation methods, the uncertainties, as pointed out by Dyrud et al. (2008), arise from the characteristics of different radar meteor observation techniques.

The cumulative mass distribution of objects crossing the Earth orbit (see Fig. 2) increases towards smaller sizes roughly as a power law. For instance Ceplecha et al. (1998)derived a flux curve based on compiled data from meteor observations, dust in-situ measurements from spacecraft, analyses of lunar craters, as well as near-Earth asteroid observations. 
Table 2.3_1 The mass range of dust and meteor observations in the ionosphere and near Earth space, the given mass ranges of different observations are discussed in the text.

\begin{tabular}{lll}
\hline Observation & Detected species & Object mass / size \\
\hline UV photometers on rockets & scattered sunlight & size $>40 \mathrm{~nm}$ \\
Ionospheric dust detectors & dust surface charges & $10^{-24}-10^{-23} \mathrm{~kg}$ \\
Plasma wave instruments & impact generated charges & $<10^{-20}-10^{-12} \mathrm{~kg}$ \\
Space dust detectors & impact generated charges & $10^{-18}-10^{-12} \mathrm{~kg}$ \\
Head echo observations & free electrons & $10^{-9}-10^{-6} \mathrm{~kg}$ \\
Radar meteor observations & free electrons & $10^{-12}-10^{-5} \mathrm{~kg}$ \\
Optical meteor observations & meteoroid \& atmospheric molecules, atoms \& ions & $10^{-8} \mathrm{~kg}-10^{-1} \mathrm{~kg}$ \\
Fireballs observations & meteoroid \& atmospheric molecules, atoms \& ions & $10^{-1} \mathrm{~kg}-10^{3} \mathrm{~kg}$ \\
\hline
\end{tabular}

The derived average flux over 100 years of meteoroids with masses $<10^{8} \mathrm{~kg}$ is $2.4 \cdot 10^{7} \mathrm{~kg}$ $\mathrm{yr}^{-1}$, with masses $<10^{-2} \mathrm{~kg}$ is $4.2 \cdot 10^{6} \mathrm{~kg} \mathrm{yr}^{-1}$ and with masses $<10^{-7} \mathrm{~kg}$ is $4 \cdot 10^{6} \mathrm{~kg}$ $\mathrm{yr}^{-1}$. An analysis of impact craters collected during an exposure experiment on a nearEarth satellite Long Duration Exposure Facility in the 1980s leads to a larger flux in the size range 20 to $400 \mu \mathrm{m}$ (approximate mass range $10^{-12}-10^{-7} \mathrm{~kg}$ ) (Love and Brownlee 1993). Analysis of marine isotope records leads to a long term average accretion rate over the last 80 million years of $3.7 \cdot 10^{7} \mathrm{~kg} \mathrm{yr}^{-1}$ (Peucker-Ehrenbrink 1996). We follow here the curve of Ceplecha et al., since it covers a broad size interval as well as observations of the past decades and assume that the flux amounts to 10 ton/day or more locally and temporarily.

Note that the mass distribution of objects smaller than $100 \mathrm{~g}$ peaks at roughly $10^{-8}$ $\mathrm{kg}$ (see Fig. 3 in Grün et al. 1985 ), implying that radar observations cover that interval in the dust and meteoroids population that causes most of the mass deposition into the atmosphere. Dust particles in the approximate size interval 1 to $100 \mu \mathrm{m}$ are observed by the thermal emission brightness and scattered light brightness in the Zodiacal light. They are well determined near the ecliptic between 0.3 and 1.7 AU distance from the Sun; the Zodiacal light is stable in time (cf. Leinert et al. 1998). In-situ measurements from spacecraft suggest that the fluxes are stable in time for dust above 1/10 micrometer size (cf. Krüger et al. 2006). In contrast to that the flux of nano dust is highly time variable (see 4.3).

Dust composition is inferred from the composition of its parentbodies, from the composition of interplanetary dust collected in the Earth atmosphere, from the analysis of micrometeorites and meteorites collected on Earth, and from optical properties derived from Zodiacal light observations. Collected samples provide compositional information and show that most particles are heterogeneous in material composition and structure and that a significant fraction of dust is porous (see e.g. Rietmeijer 2002). For a rough estimate of the materials enter the Earth atmosphere, we assume that the majority of entering objects have cometary dust composition, similar to a local interstellar cloud dust model that was derived from astronomical observations and space measurements (Kimura et al. 2003). This model assumes that the elements are contained in the typical cosmic materials corundum $\left(\mathrm{Al}_{2} \mathrm{O}_{3}\right)$, kamacite (FeNi), forsterite $\left(\mathrm{Mg}_{2} \mathrm{SiO}_{4}\right)$, enstatite $\left(\mathrm{MgSiO}_{3}\right)$, troilite $(\mathrm{FeS})$ and organic refractory compounds. Following the element abundances assumed in this model the mass percentages of major elements deposited into the ionosphere are: carbon $25 \%$, nitrogen $4.5 \%$, oxygen $38 \%$, magnesium $7.1 \%$, aluminium $<1 \%$, silicon $7.6 \%$, sulphur $3.2 \%$ and iron $13 \%$. The amount of hydrogen and noble gases in cosmic dust and meteoroids is not well determined. 


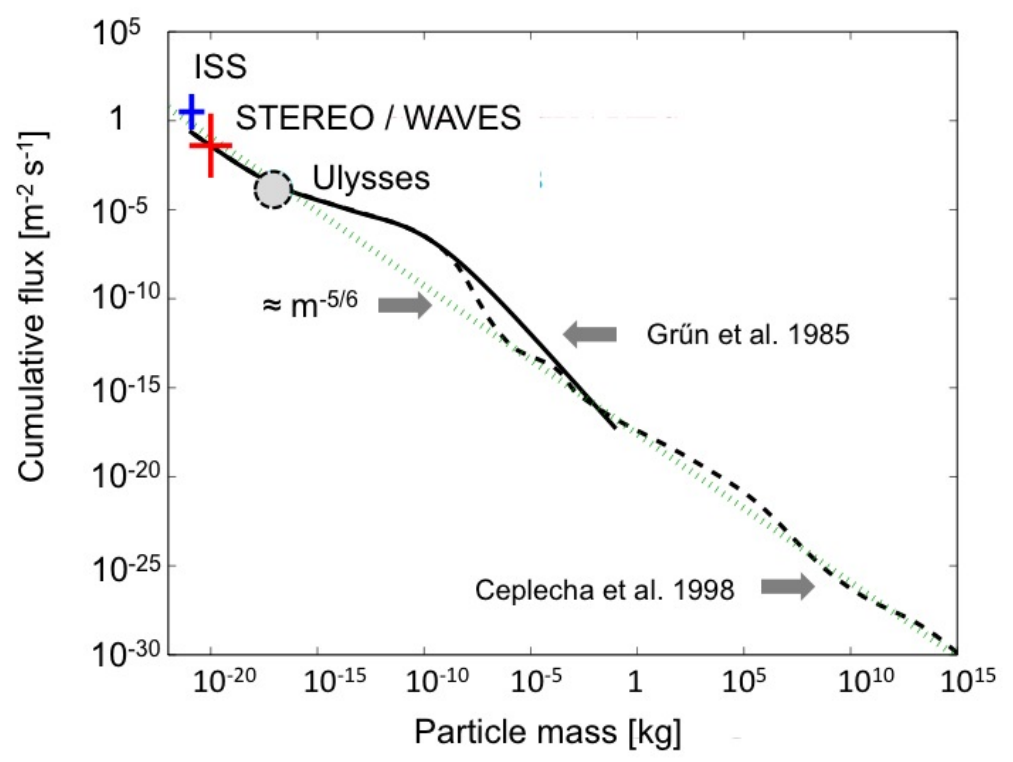

Fig. 2 The flux of dust with mass greater or equal the given mass near 1 AU. Shown are the interplanetary dust flux model that is derived primarily from dust in-situ measurements and analysis of lunar microcraters (solid line, (Grün et al. 1985)) and the meteor model that also includes the small solar system objects (dashed, (Ceplecha et al. 1998)). The dotted line describes a flux $\sim m^{-5 / 6}$ from collisional fragmentation equilibrium (Dohnanyi 1978). The flux values derived from analysis of impact craters on the International Space Station (ISS, Carpenter et al. 2005), from measurements of the Ulysses dust instrument (Wehry and Mann 1999) and and from STEREO plasma wave measurements during the year 2007 are shown. This figure is adapted from Meyer-Vernet et al. (2009b).

\subsection{Dust plasma interactions}

Dust can interact with a plasma in various ways. Dust can act as a sink of plasma particles by absorbing plasma species. Dust can also act as a source of electrons, via processes such as photoemission due to solar ultraviolet radiation (see section 5.1). The ablation of heated dust or meteors can release dust and atomic/molecular species into the plasma (see section 3.1). Impinging particles are decelerated and undergo charge exchange or may remove atoms or ions from the dust (i.e. sputtering) (see section 4.1).

Dust in a plasma and radiative environment is generally electrically charged owing to various processes, such as the collection of plasma particles, photoemission, secondary emission in the presence of high energy plasma particles, etc. (see section 5.1). When the spacing between dust particles is much larger than the Debye length, the charged dust particles are shielded out to such an extent that the electrostatic interaction between dust particles is negligible. On the other hand, when the spacing between dust particles is smaller than or comparable to the Debye length, the electrostatic interaction between dust particles is important. In this case the dust possibly gives rise to collective effects in the "dusty plasma", see reviews by Mendis and Rosenberg 1994; Fortov et al. 2005; Shukla and Mamun 2002).

Both theoretical and laboratory experimental studies show that the presence of charged dust can modify known plasma waves and instabilities and give rise to new very low frequency waves and instabilities associated with the massive dust grains (for reviews see e.g. 
Table 2.4 2 Parameters of dust and gas in the interplanetary medium near Earth orbit and in the ionosphere. Dust masses $m<10^{-13} \mathrm{~kg}$ correspond to approximate sizes $<2 \mu \mathrm{m}$, dust masses $m<10^{-20} \mathrm{~kg}$ to sizes $<10 \mathrm{~nm}$. Basic solar wind parameters are from Allen's Astrophysical Quantities (Allen 1976). The density of nano dust near $1 \mathrm{AU}$ is estimated assuming the flux $\phi=0.6 \mathrm{~m}^{-2} \mathrm{~s}^{-1}$ and velocity $300 \mathrm{~km} / \mathrm{s}$ for dust with mass $10^{-20} \mathrm{~kg}$, the number densities of larger dust are from Mann et al. (2004). The ionospheric densities, temperatures and ionization fractions are discussed in Section (2.2). Numbers vary significantly and these are therefore rough estimates. The dust densities in the ionosphere are from the observations cited in this text (see Section 3.7 and Fig.5)

\begin{tabular}{lll}
\hline & Interplanetary Medium & Earth Ionosphere \\
\hline & Medium & Ionosphere \\
\hline $\mathrm{M}_{\text {gas }} / \mathrm{kg} \mathrm{m}^{-3}$ & $\approx 0.1-1 \times 10^{-19}$ & $>5 \times 10^{-16}$ \\
$\mathrm{M}_{\text {dust }} / \mathrm{kg} \mathrm{m}^{-3}$ & $2 \times 10^{-18}$ & $($ not measured $)$ \\
$\mathrm{n}_{\text {dust }} / \mathrm{m}^{-3}\left(m<10^{-13} \mathrm{~kg}\right)$ & $1 \times 10^{-7}$ & $($ not measured $)$ \\
$\mathrm{n}_{\text {dust }} / \mathrm{m}^{-3}\left(m<10^{-20} \mathrm{~kg}\right)$ & $\approx 10^{-6}$ & $\left(10^{6}-10^{9}\right)$ \\
$\mathrm{n}_{\mathrm{e}} / \mathrm{n}_{\mathrm{n}}$ & $\approx 1$ & $\approx 10^{-10}-10^{-3}$ \\
$\mathrm{n}_{\mathrm{e}} / \mathrm{m}^{-3}$ & $5 \times 10^{6}$ & $10^{9}-10^{15}$ \\
$\mathrm{~T}_{\mathrm{e}} / \mathrm{K}$ & $2 \times 10^{5}$ & $<1500$ \\
\hline
\end{tabular}

Merlino et al, 1998; Verheest 2000, Shukla and Mamun, 2002; Fortov et al., 2005; Shukla and Eliasson, 2009).

For example, dust can affect standard waves and instabilities through effects on equilibrium charge neutrality, given by $n_{e}+\varepsilon Z_{d} n_{d}=n_{i}$, where $Z_{d}$ is the dust charge state, $\varepsilon=1(-1)$ for negatively (positively) charged dust, and $n_{d}, n_{e}, n_{i}$ are the dust, electron and ion densities, respectively.

The phase speed of ion acoustic waves, which is $\propto\left(n_{i} / n_{e}\right)^{1 / 2}$, increases in the presence of negatively charged dust, so that the waves undergo less Landau damping and can be more easily excited (Shukla and Silin 1992; D’Angelo 1990; Rosenberg 1993). As another example, the motion of charged dust leads to a new very low frequency dust acoustic wave, where the dust provides the inertia and the electrons and ions provide the pressure to sustain the wave (Rao et al, 1990). Dust acoustic waves have phase speed less than the ion thermal speed and can be driven unstable by the streaming of ions (or electrons) with small speeds relative to the dust (Rosenberg, 1993). Although potentially interesting, the application of possible instabilities to near-Earth dusty plasmas is in a relatively early stage (see section 3.8).

\section{Dust in the ionosphere}

Entrance of large enough particles of extraterrestrial matter into the Earth' $s$ atmosphere is associated with light emission and ionization phenomena giving rise to meteors. A region of ionized gas forms near the meteoroid and expands almost instantaneously. The head moves with the speed of the meteoroid. It is followed by the meteor trail (also called train), that extends behind the meteoroid body. At typical heights between 80 and $120 \mathrm{~km}$, both head and trail, depending on meteoroid mass and entry conditions, can be observed optically as well as by radar backscattering. The relation between observed brightness and ablated meteoroid mass varies with the atmospheric conditions and with meteoroid parameters like altitude, entry speed, size and composition. Because of this variation physical parameters 
like the bulk density can not be inferred completely from the observations. Meteoric smoke particles form from the ablated meteoroid material and are detected in-situ from sounding rockets. Charged dust particles are suspected to influence some meteor observations and be observed by radar scattered signals, but distinguishing between the heavy ion and charged dust signals is still a challenge. Observations of dusty plasma effects are still limited. Our discussion is primarily based on meteor phenomena and we refer the reader to detailed reviews of the particle interactions in the PMSE region (e.g. Cho and Röttger 1997, Rapp and Lübken 2004).

We start by describing the classical ablation process by evaporation (3.1). This explains most radar meteors (section 3.2), and optical meteors (see 3.3). High altitude meteors are discussed in section 3.4. They are associated to non-thermal ablation (3.5) especially due to sputtering. Fragmentation events, as described in section 3.6 limit the interpretation of meteor observations. The section 3.7 is devoted to atmospheric dust measurements and section 3.8 elaborates the possibility of dust-plasma instabilities forming in the Earth's ionosphere.

\subsection{Meteoroid ablation by evaporation}

Meteoroids entering the Earth' s atmosphere lose mass mostly as a result of vapor production. The intensity of radiation $I$ and the linear electron concentration $\alpha$ (i.e. number of free electrons produced by evaporated meteoroid atoms per meter), given in the following analytical expressions, can both be observed in the meteor phenomena:

$$
I=-\tau\left(\frac{v^{2}}{2} \frac{d m}{d t}+m v \frac{d v}{d t}\right) \quad \alpha=-\frac{\beta}{\mu v} \frac{d m}{d t}
$$

where $v, m$ are the meteoroid velocity and mass, $\tau$ the luminous efficiency, $\beta$ the ionizing efficiency, and $\mu$ is the average mass of the ablated atoms (Ceplecha et al. 1998). The meteoroids size relation to the atmospheric free path at different altitudes corrected for meteoroid velocity defines the regime of interaction with ionosphere and the character of ablation (Bronshten 1983; Popova 2004). Large meteoroids lose most of their mass in the continuum flow regime whereas small meteoroids, discussed in this paper, interact with ionosphere mainly in the free molecular flow or transition flow regimes. (Note that, since the difference between thermal velocity and meteoroid velocity influences the physics of the interaction process, these boundaries are dependent both on size and velocity of meteoroid). The screening of meteoroid surface by evaporated material and subsequent decrease of the heat transfer coefficient must be taken into account in the transition flow condition modeling (Popova 2004 and references therein).

Momentum transfer in collisions between atmospheric gases and meteoroids leads to heating, followed by ablations and thermal radiation, the latter effects causing the meteoroid to cool. At a given altitude the behavior of a particle penetrating the ionosphere is determined by its size, velocity and material properties and this is shown in Fig. 3. Based on meteor physics equations analysis similar to that introduced the 1960s (see e.g. Popova 2004 and references therein), it shows that for impact speed of $40 \mathrm{~km} / \mathrm{s}$, stony objects $\left(\leq 10^{-6} \mathrm{~m}\right)$, decelerate before being substantially heated and the heating of objects with radiua $\mathrm{R} \leq$ $10^{-4} \mathrm{~m}$ is limited by thermal radiation. Larger particles evaporate almost totally at altitudes between 100 and $80 \mathrm{~km}$.

The recent analysis of micrometeoroids collected on the arctic surface snow point to mechanical destruction and weathering (Duprat et al. 2007). Rietmeijer (2002) points out that even certain classes of the interplanetary dust particles that are collected - almost intact 
- in the Earth atmosphere show traces of flash heating to 300 - 1000 degree Celsius. Organic material may survive the entry process in the small decelerated particles which were not heated up to high temperatures (see e.g. Coulson 2002, Coulson and Wickramasinghe 2003). Besides, the more volatile metals may sublimate earlier during the meteoroid entry (differential ablation, e.g. McNeil et al., 1998). The heating of larger objects ( $\left.\mathrm{R}>10^{-3} \mathrm{~m}\right)$ cannot be characterized by uniform temperature and the heated layer is about $10^{-4}-10^{-3}$ $\mathrm{m}$ in thickness (Bronshten 1983; Ceplecha et al. 1998).

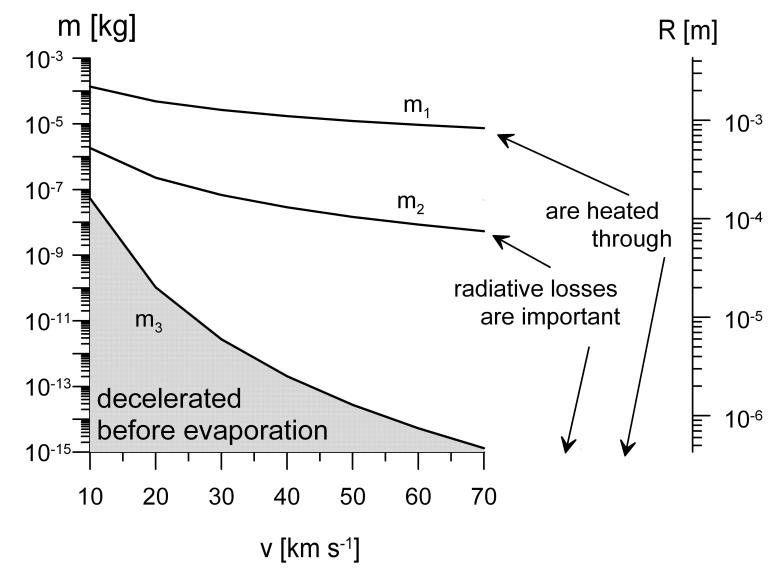

Fig. 3 Rough mass and size boundaries demonstrating importance of different processes.

The energy flux on the meteoroid increases as the body reaches denser regions of the atmosphere. Intensive evaporation starts when the temperature rise up to about $2000-2500$ $\mathrm{K}$, which occurs at about $110-130 \mathrm{~km}$ for porous bodies with $\mathrm{R} \sim 10^{-3}-10^{-1} \mathrm{~m}$ (Bronshten 1983; Ceplecha et al. 1998). Below this altitude the incoming energy contributes to ablation mainly. Above a certain size limit, which is dependent on material properties and velocity, the entering objects do not fully ablate (roughly objects larger 1-10 cm for low velocity entry). The remnant objects continue a dark flight and eventually reach the surface of the Earth as meteorites after undergoing a variety of heterogeneous reactions. The strongly temperature related meteoroid mass loss processes are generally called thermal ablation and other processes are usually excluded from the consideration. Aside from the thermal ablation, dedicated fragmentation models and the different conditions needed for high altitude meteors are discussed below.

\subsection{Radar meteor observations}

The meteor trail echoes are radio waves scattered by free electrons in the trail that extends for meters to kilometers behind the meteoroid. They last from tenths of seconds to minutes and dominate measurements by specular meteor radars (SMR) such as AMOR and CMOR (see Pellinen-Wannberg et al. 2008, for an overview of radar facilities).

According to their distinctly different shapes of the radar signal, trails are denoted as over-dense and under-dense trails. In the case of an under-dense meteor trail the plasma frequency is below the radar frequency. The scattered signals result from individual free 
electrons within the trail and are reinforced due to constructive interference if the trail is oriented perpendicularly to the radar beam. Since the beam is wide, many trails fulfill the perpendicularity condition. When the plasma (electron) density is high, so that the plasma frequency exceeds the radar frequency, the radio waves can not penetrate the trail, which then forms a reflecting obstacle to the radar beam. This case is denoted as over-dense trail. The over-dense echo power is approximately constant until the expanding trail reaches the underdense condition, i.e. the column electron density along the beam is $<10^{14} \mathrm{~m}^{-1}$ (McKinley 1961) (the exact value depending on the radar frequency).

Theoretical predictions of the initial radius of evolving plasma column lead to differing results. Experimentally derived values range from several $10 \mathrm{~cm}$ to about $3 \mathrm{~m}$ depending on altitude and meteoroid velocity (Ceplecha et al. 1998). The initial radius (similarly as the mean free path of atmospheric species) increases with altitude. Hence, the phase difference between the scattered signals increases. As soon as the trail extends to more than half the radar wavelength constructive interference ceases, signals interfere destructively and the measured reflected power decreases exponentially. Hence the reflective properties of the meteor trail and the surrounding ionosphere for a given radio frequency/wavelength limit the height of SMR observations. This is called the radio meteor ceiling effect.

Head echoes originate from the plasma in the immediate surrounding of the meteoroid and detect the submillimeter-size meteoroid population in the $10^{-9}$ to $10^{-6} \mathrm{~kg}$ mass range. They are transient, typically lasting for less than tens of milliseconds, and characterised by high Doppler shifts. Only the most powerful SMRs are sensitive enough to observe such echoes and 700 head echoes were reported during 25 years of data on different showers (Jones and Webster 1991). Head echoes dominate measurements of High Power Large Aperture radars (HPLA), such as ALTAIR, Arecibo, and EISCAT (Pellinen-Wannberg et al. 2008), and they were firstly studied with EISCAT in 1990 (Pellinen-Wannberg and Wannberg 1994). The most powerful HPLA radars record 700 head echoes of sporadic meteors within a quarter of an hour (Close et al. 2000).

With head echo observations, parameters such as velocity and deceleration components along the radar beam, altitude of occurrence and rates can be obtained directly (Kero et al. 2008a). Tristatic measurements allow observing fragment formation (Kero et al. 2008b) and estimating the meteoroid mass and size with high accuracy (Kero et al. 2008c). These measurements also lead to a better derivation of the magnitude and orbit determinations for individual meteors (Szasz et al. 2007; Szasz et al. 2008). Janches et al. (2009) even suggest that head echo observations allow observing differential ablation. Observations of a single head echo at two or even three frequencies are rare, but allow closer studies of the head echo character (Wannberg et al. 1996; Close et al. 2002; Pellinen-Wannberg 2004).

\subsection{Optical meteor observations}

The most common optical meteor phenomena, their formation mechanisms and the main conditions of appearance are listed in Table 3.3_1 (from Mann 2009). The approximate mass ranges are for optical meteors $10^{-8}<m<10^{-1} \mathrm{~kg}$ and for fireballs $10^{-1}<m<10^{3} \mathrm{~kg}$. The immediate surrounding of the meteoroid body displays the brightest part of the meteor, the head. Behind the head, different types of trails are observed and arise from different physical processes. The listed trail phenomena were discussed in detail by (Borovička 2006). Meteor spectra reveal mostly emission lines as well as some molecular bands; thermal continuum radiation is typically negligible and only dominant in the fireball spectra (e.g. Borovičcka 1999). Spectra change along meteor trajectory. The emission is due mostly to neutral atoms 
Table 3.3_1 The major optical meteor phenomena. This table is adapted from Mann (2009), it basis on a detailed discussion of the trail phenomena by Borovička (2006).

\begin{tabular}{|c|c|c|c|}
\hline Type & Mechanism(s) & Phenomenon & $\begin{array}{l}\text { Main conditions of appear- } \\
\text { ance }\end{array}$ \\
\hline head & $\begin{array}{l}\text { instantaneous line emis- } \\
\text { sion of meteoroid and } \\
\text { atmospheric species sur- } \\
\text { rounding the meteoroid }\end{array}$ & $\begin{array}{l}\text { major meteor brightness } \\
\text { that lasts for about a sec- } \\
\text { ond }\end{array}$ & $\begin{array}{l}\text { altitude between } 120 \text { and } \\
80 \mathrm{~km}(20 \mathrm{~km} \text { for fireballs) }\end{array}$ \\
\hline flare & $\begin{array}{l}\text { sudden fragmentation or } \\
\text { change of physical param- } \\
\text { eters }\end{array}$ & $\begin{array}{l}\text { sudden increase of meteor } \\
\text { brightness by more than } \\
\text { one magnitude }\end{array}$ & $\begin{array}{l}\text { mostly for high velocity } \\
\text { meteors }\end{array}$ \\
\hline \multicolumn{4}{|l|}{ trail } \\
\hline $\begin{array}{l}\text { gaseous } \\
\text { wake }\end{array}$ & $\begin{array}{l}\text { line emission of rarefied } \\
\text { non-equilibrium gas that } \\
\text { follows the head }\end{array}$ & $\begin{array}{l}\text { emission spectrum similar, } \\
\text { but not identical to the } \\
\text { head evolves } 100 \text { s of me- } \\
\text { ters to several kilometers } \\
\text { behind the head }\end{array}$ & altitude beyond $55 \mathrm{~km}$ \\
\hline $\begin{array}{l}\text { particu- } \\
\text { late } \\
\text { wake }\end{array}$ & $\begin{array}{l}\text { individual meteor heads } \\
\text { formed around fragments } \\
\text { released from the mete- } \\
\text { oroid }\end{array}$ & $\begin{array}{l}\text { emission spectrum similar } \\
\text { to meteor head }\end{array}$ & $\begin{array}{l}\text { below the altitude of frag- } \\
\text { mentation (mostly at low } \\
\text { altitude) }\end{array}$ \\
\hline $\begin{array}{l}\text { green } \\
\text { train }\end{array}$ & $\begin{array}{l}\text { reactions among atmo- } \\
\text { spheric species }\end{array}$ & $\begin{array}{l}\text { OI } 557.7 \mathrm{~nm} \text { (forbidden } \\
\text { line) emission occuring } \\
\text { from } 1 \text { to } 2 \mathrm{~s} \text { after meteor } \\
\text { disappearance }\end{array}$ & $\begin{array}{l}\text { mostly for high velocity } \\
\text { meteors; height profile dif- } \\
\text { ferent from that of the me- } \\
\text { teor }\end{array}$ \\
\hline $\begin{array}{l}\text { persistent } \\
\text { train }\end{array}$ & $\begin{array}{l}\text { atomic recombination } \\
\text { and/or chemiluminescence } \\
\text { of cooling rarefied gas }\end{array}$ & $\begin{array}{l}\text { visible for tens of min- } \\
\text { utes after meteor disap- } \\
\text { pearance; consist of after- } \\
\text { glow phase, recombination } \\
\text { phase and continuum }\end{array}$ & $\begin{array}{l}\text { bright fireballs } 75 \text { to } 100 \\
\mathrm{~km} \text { altitude }\end{array}$ \\
\hline $\begin{array}{l}\text { reflection } \\
\text { train }\end{array}$ & $\begin{array}{l}\text { Sunlight scattered at frag- } \\
\text { ment dust particles gener- } \\
\text { ated by fireballs }\end{array}$ & $\begin{array}{l}\text { bright spectral continuum } \\
\text { and band emission of } \\
\text { metal oxides that lasts for } \\
\text { hours }\end{array}$ & $\begin{array}{l}\text { form at any height; ob- } \\
\text { served during daytime or } \\
\text { twilight }\end{array}$ \\
\hline
\end{tabular}

and singly charged atomic ions (see Borovička 1993 for a compilation of the observed lines). The observed spectra are empirically described by two gas components with temperature $\sim 4000 \mathrm{~K}$ for the main component and $\sim 10000 \mathrm{~K}$ for a second component. This model matches most observed spectra. In a typical application of this model, the emission lines of the main component are used to derive the chemical composition of the entering meteoroids (Borovička 1993). This empirical model, however, uses simplified description of the luminous volume and doesn't take into account possible deviation from thermodynamical equilibrium. Moreover the observed line emission partly originates from atmospheric, as opposed to meteoroid species (Campbell-Brown 2004; Hawkes 2002; Lindblad et al. 2003).

\subsection{High altitude meteors}

According to the scenario outlined above, meteor ablation starts below $130 \mathrm{~km}$. Indeed, among more than 4500 photographic meteors recorded in the Meteor Data Center of the International Astronomical Union (Lindblad et al. 2003) only $0.1 \%$ (five cases) had beginning 
heights above $130 \mathrm{~km}$ and average beginning heights are below $100 \mathrm{~km}$ (Koten et al. 2006). Beginning heights increase with entry velocities. Indeed Campbell et al. (2000) derived a mean beginning height of $112 \mathrm{~km}$ for Leonids $(\mathrm{v} \sim 72 \mathrm{~km} / \mathrm{s}$ ) with average mass of 1.4 . $10^{-6} \mathrm{~kg}$ in 1998 (79 meteors in the sample). Brown et al. (2002) found that the ablation zone begun at about $123 \mathrm{~km}$ altitude for meteors with average mass of $\sim 10^{-6}-10^{-7} \mathrm{~kg}$ in 1999 (232 Leonids).

Also, high altitude meteors, i.e. those observed beyond classical ablation heights, are often observed for meteoroids with high entry velocity. Koten et al. (2006) found about 5\% high-altitude meteors, with beginning heights between roughly 130 and $150 \mathrm{~km}$. among 164 observed shower meteors. But not a single Orionid was detected above $130 \mathrm{~km}$ despite their having a high entry velocity $(\mathrm{v} \sim 66.5 \mathrm{~km} / \mathrm{s}$ ) The beginning height obviously varies with the spectral range and the sensitivity of the observation device. Simultaneous observations of two high-altitude Leonids in 1995 and 1996 showed beginning heights below $130 \mathrm{~km}$ for the photographic observations and around $160 \mathrm{~km}$ for intensified video observations (Fujiwara et al. 1998). Studying the high altitude spectra of 8 Leonids Spurný et al. (2004) find that the high altitude signal is mainly due to emission of the oxygen line at $777 \mathrm{~nm}$ together with a faint continuum that they suggest to be due to $\mathrm{N}_{2}$ molecular bands.

Another Leonid observation from 2002 shows a trail starting at $145 \mathrm{~km}$ altitude (PellinenWannberg et al. 2004). It was detected with the optical auroral imaging network ALIS (Brändström 2003) through two filters at $589.3 \pm 10 \mathrm{~nm}$ in the range of a sodium emission line $(\mathrm{NaI})$ and at $422.7 \pm 14 \mathrm{~nm}$ in the range of calcium line $(\mathrm{CaI})$. While the sodium trail started at $110 \mathrm{~km}$, the signal through the calcium filter started at 145 altitude, was transient and very strong (Fig. 4). In addition to calcium this filter covered emission lines from iron and the hydrogen $\gamma$ Balmer series. Pellinen-Wannberg et al. (2004) suggested that since calcium and iron lines were expected to be weaker, the emission originated from the $\gamma$ Balmer series of a hydrogen compound induced by hypervelocity collisions.

Pellinen-Wannberg and Wannberg (1994) reported the detection of radar meteor trails in 1990 up $160 \mathrm{~km}$ altitude and later suggested that heavy meteoric ions modify the incoherent scatter spectrum from these trails (Pellinen-Wannberg and Wannberg 1996). During the 1998 Leonids Brosch et al. (2001) monitored echoes from meteors up to $400 \mathrm{~km}$ altitudes. They have interpreted the origin of these trails as sputtering of atmospheric particles on meteoroids at these altitudes.

Brosch et al. (2004) presented observations of simultaneous radar and optical Leonids and among that showed a radar event at very high altitude $(\sim 250 \mathrm{~km})$, with a sequence of echoes indicating a sinuous path of the radar reflector. It was hypothesized that the radar scattering might occur from charged dust clouds, whose diffusion would be much smaller than ions owing to the large dust mass, and which could be carried by high-altitude winds. If dust is involved, one may surmise that it might have to do with the possible formation and persistence of electron density gradients owing to the presence of negatively charged dust (Kelley et al. 1998). For detection with L-band radar at $\sim 1 \mathrm{Ghz}$, the Bragg condition for radar backscatter requires density irregularities with scale size $\sim 1.5 \cdot 10^{-1} \mathrm{~m}$.

The very high altitude trail observations with radar reported by Pellinen-Wannberg and Wannberg $(1994 ; 1996)$ and Brosch et al. $(2001$; 2004) were performed during or close to solar cycle maximum conditions (1990 and 1999), during which the atmospheric and ionospheric densities usually expand to higher altitudes (Fig. 1). The possible connection between high altitude trails and solar cycle should be carefully studied during the approach of the next solar maximum within the coming years (Pellinen-Wannberg et al. 2010). 


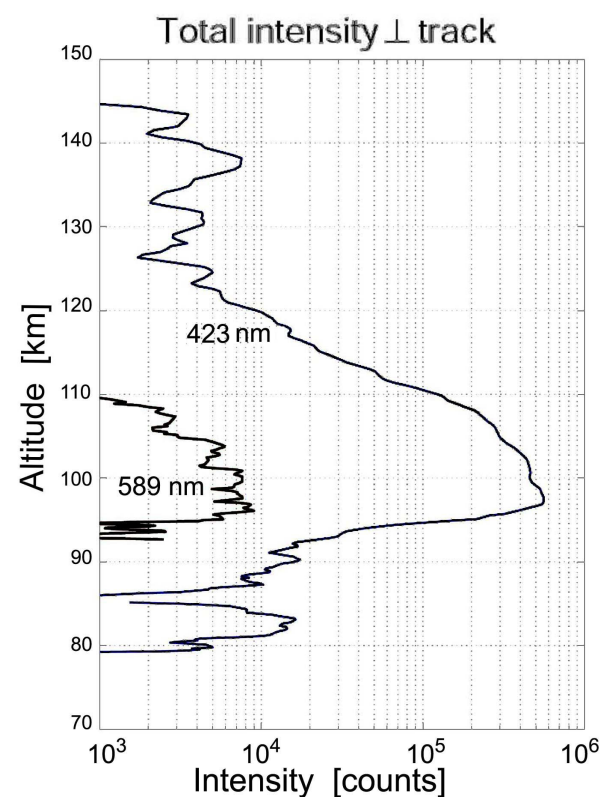

Fig. 4 Two optical altitude profiles of the same meteor through a $589 \mathrm{~nm}$ filter and a $423 \mathrm{~nm}$ filter. While the profile at $589 \mathrm{~nm}$ is quite typical for the usually quite strong sodium emission, the profile measured with the $423 \mathrm{~nm}$ filter starts at much higher altitude and is much stronger than expected for calcium or iron emissions within the filter bandwidth. The line is suggested to originate from the $\mathrm{H}_{\gamma}$ line at $433 \mathrm{~nm}$ associated with strong volatile $\mathrm{H}_{2} \mathrm{O}$ gas emission (Pellinen-Wannberg et al. 2004).

\subsection{Non-thermal ablation}

Sputtered particles may cause formation of ionized meteor trails recorded by radars and may produce observable (high altitude) meteors, even if the mass loss due to sputtering is small. Sputtering is the only ablation mechanism for small particles, which are decelerated before being substantially heated. The sputtering of meteoroid material and the scattering of atmospheric species at meteoroids are connected to the formation of high altitude meteors (section 3.4). Sputtering generally occurs for impacting ions, but also for other particles (for instance neutral atoms, molecules) and it occurs over a large range of energies from sub $\mathrm{keV}$ to $\mathrm{MeV}$ (we refer the reader to Behrisch and Eckstein 2007). A couple of percent of the sputtered species are ions and the removed atoms or ions are not necessarily in their ground states.

Popova et al. (2007) have suggested that the oxygen luminosity observed at high altitudes can be generated by the interaction of the sputtered atoms and ions with the background ionosphere (several percent of sputtered species are ionized). The sputtered atoms and ions typically have kinetic energy more than 1000 times the energy of surrounding ionospheric particles. When thermalizing through collisions they generate the luminosity. This is shown by Vinkovic (2007) in a model that also predicts the spatial extension of the generated luminosity. Rosenberg (2008) suggests that fast ions sputtered from a meteoroid might generate waves in the background plasma possibly detectable by radar scattering. 
The sputtering models used in the studies mentioned above have limitations. The conditions for sputtering of meteoroids entering the atmosphere are determined by the atmospheric density (see section 2 ) and the entry speed. Assuming a maximum meteoroid entry speed of $72 \mathrm{~km} / \mathrm{s}$ and impact of atmospheric $O^{+}$one obtains a maximum energy of ions of $430 \mathrm{eV}$, corresponding to $27 \mathrm{eV}$ per nucleon. The major species impinging on the entering meteoroids are $\mathrm{O}, \mathrm{O}_{2}$ and $\mathrm{N}_{2}$. For meteoroids with entry velocity smaller than about $40 \mathrm{~km} / \mathrm{s}$ energies of impinging atmospheric species are close to the threshold energies for sputtering and those are in most cases determined with great uncertainty.The sputtering yield at sub - keV energies can be influenced by many processes. Precise composition and structure of meteoroids as well the state of their surfaces are unknown. There appears to be some experimental evidence that sputtering from oxygen covered surfaces of various elements can produce high ion fraction (see Fig. 2 in Krauss and Grün 1979). The meteoroid surface may indeed be oxygen covered, since it is traveling through the ionosphere. Also atmospheric species may react at and be scattered from the meteoroid surface.

Opposite to what was recently suggested by Rogers et al. (2005) and Hill et al. (2005), sputtering does not cause significant mass loss. Kero (2008) reconsidered the mass loss using a numerical model of sputtering and thermal ablation similar to that used by the former authors. He suggested that the atmospheric densities in the Rogers et al. (2005) paper are a factor of 100 too high and that this led the authors to overestimate the mass loss caused by sputtering (note that the atmospheric densities are not explicitly given in the Rogers et al. (2005) paper). Kero found that for a $10^{-6} \mathrm{~kg}$ test particle with initial velocity $60 \mathrm{~km} / \mathrm{s}$ sputtering of meteoroid mass is negligible compared to (conventional) ablation. Vondrak et al. (2008), based on another ablation model, find that sputtering only causes significant mass loss (35 percent at maximum) for the smallest meteoroids $\left(<10^{-14} \mathrm{~kg}\right)$ with high initial velocities $\left(<45 \mathrm{~km} \mathrm{~s}^{-1}\right)$.

\subsection{Fragmentation events}

Numerous observed meteor phenomena are thought to result from fragmentation events. Without considering fragmentation, the meteoroid masses estimated based on meteoroid deceleration (dynamic mass) are largely different from those derived from the observed radiated energy (photometric mass). Radar reflections from meteor trails often differ from the predictions of simple models. Fragmentation also causes interference patterns in head echoes.

Currently two basic approaches are followed to describe fragmentation.The dust ball model assumes that meteoroids consist of individual dust particles that are released during fragmentation (Hawkes and Jones 1975). The Quasi-continuous fragmentation model (QCF) assumes a gradual release of the smallest fragments from the surface of a parent meteoroid and their subsequent evaporation, without specifying the fragmentation mechanisms (Bronshten 1983).

Hawkes et al. (2004) described high (spatial and temporal) resolution optical observations with the dust ball model and suggested that fragmentation occurred for at least half of the studied very faint meteors. Also light curves of faint Leonids $\left(\sim 10^{-7}-10^{-4} \mathrm{~kg}\right)$ and other meteor showers are quite reasonably explained in the frame of the dustball model (Koten et al. 2004). An ablation model for faint meteors that included fragmentation similar to the dust ball model was elaborated by Campbell-Brown and Koschny (2004). CampbellBown and Close (2007) further modified the same ablation model to calculate ionization densities; they studied 25 head echoes in detail and found that most of the observations 
are consistent with meteoroid fragmentation, though their was no evidence for large spatial separation of the fragments.

Some of the observations of fragmentation events suggest that there is no large separation between fragments and a large fraction of meteor phenomena can be reasonably reproduced without taking fragmentation into account, Considering fragmentation is nevertheless important for analyzing the observations, since the derived material density of the meteoroid depends strongly on assuming or not assuming fragmentation (Bellot Rubio et al. 2002). Babadzhanov (2002) successfully applied the QCF model to 111 our of 197 observed bright photographic meteors $\left(\mathrm{m}>10^{-5} \mathrm{~kg}\right.$ ) to derive mean bulk densities of meteoroids belonging to six meteoroid streams and to the sporadic background. He points out that the derived bulk densities are in average larger by one order of magnitude than proposed previously not taking into account the fragmentation.

\subsection{Atmospheric dust measurements}

Entering meteoroids leave layers of metallic atoms and some metallic ions in the ionosphere. As reported by Krankowsky et al. (1972), the reported isotope abundances of metals seem to conform with solar isotope abundances. LIDAR measurements have measured such layers consisting of $\mathrm{Na}, \mathrm{Fe}, \mathrm{K}$, and $\mathrm{Ca}$ (McNeil et al., 2002). The metal layers are maintained in a steady-state by the equilibrium between constant dust entry followed by vaporization and removal via complex chemical reactions between the metal atoms and the atmospheric species in their descent to the ground (see Plane, 2002). Besides the neutral elements, ionic species, such as $\mathrm{Fe}^{+}, \mathrm{Mg}^{+}, \mathrm{Ca}^{+}$and other minor metallic constituents are also generated in the ablation of the dust particles. Metal ion formation from the atoms is mainly due to charge exchange reactions and to some extent due to collisional excitation, less so due to photo-ionization (see e.g. Plane, 2002).

Rosinski and Snow (1961) first introduced the idea that meteoric vapors recondense into $\mathrm{nm}$-sized particles (i.e. smoke) (corresponding to a few 100s of silicate molecules) at altitudes near $80-90 \mathrm{~km}$. Hunten et al. (1980) later calculated the evolution and fate of these particles based on the assumption that the particles condensed to sizes of 1 to several 10s of $\mathrm{nm}$; they considered the evolution of the particles taking into account coagulation, diffusion, and sedimentation. At $90 \mathrm{~km}$ altitude, the density of $20 \mathrm{~nm}$ particles thus formed exceeds that of the incoming interplanetary dust. Kalashnikova et al. (2000) calculated the formation of the meteoric smoke in a similar approach and found that the smoke formation depends on the properties of incoming micrometeoroids. The altitudes of the meteoric smoke particles (80-100 km) are also the altitudes, at which polar mesospheric summer echoes (PMSE) and noctilucent clouds occur. The former refers to the strong radar echoes at $50 \mathrm{MHz}-1.3 \mathrm{GHz}$ from the summer polar mesosphere at altitudes of 80 - $90 \mathrm{~km}$ (Czechowsky et al., 1979; Cho and Rötger 1997), while the latter are luminescent clouds observed shortly after sunset slightly above $80 \mathrm{~km}$. Both phenomena are associated with the presence of meteoric metals, which catalyze the formation of ice crystals, since at these altitudes homogeneous nucleation is too slow as a source for ice crystals. Megner et al. (2008) have shown that transport of meteoric material might affect the coagulation conditions. The composition of the meteoric smoke is also important; for example sodium bicarbonate $\left(\mathrm{NaHCO}_{3}\right)$ is particularly effective as a condensation nucleus and has been suggested to facilitate the formation of noctilucent clouds (Plane 2000).

In 1992 Schulte and Arnold reported in-situ detection of meteoric smoke particles with mass spectrometer measurements. Kelly et al (1998) reported on the simultaneous radar 
and rocket detection of a possible meteor condensation trail and meteoric dust in the upper mesosphere. The size of the dust was estimated to be $\sim 50 \mathrm{~nm}$ based on the fall speed. Since then the meteoric smoke particles have been studied by several dedicated rocket-borne experiments and experimental results are shown in Fig. 5. The detection techniques are further discussed in Section (6.1. The experimenters report the detection of both negatively - and positively - charged dust (e.g. Havnes et al. 2001; Rapp et al. 2003). In the tropical mesosphere a layer of positively charged nano-particles was found at altitudes $\sim 90-100 \mathrm{~km}$ (Gelinas et al. 1998). The concentration of these particles appears to be lower than in other experiments $\left(\sim 10^{7} \mathrm{~m}^{-3}\right)$. Particles at the bottom of the layer were charged negatively.

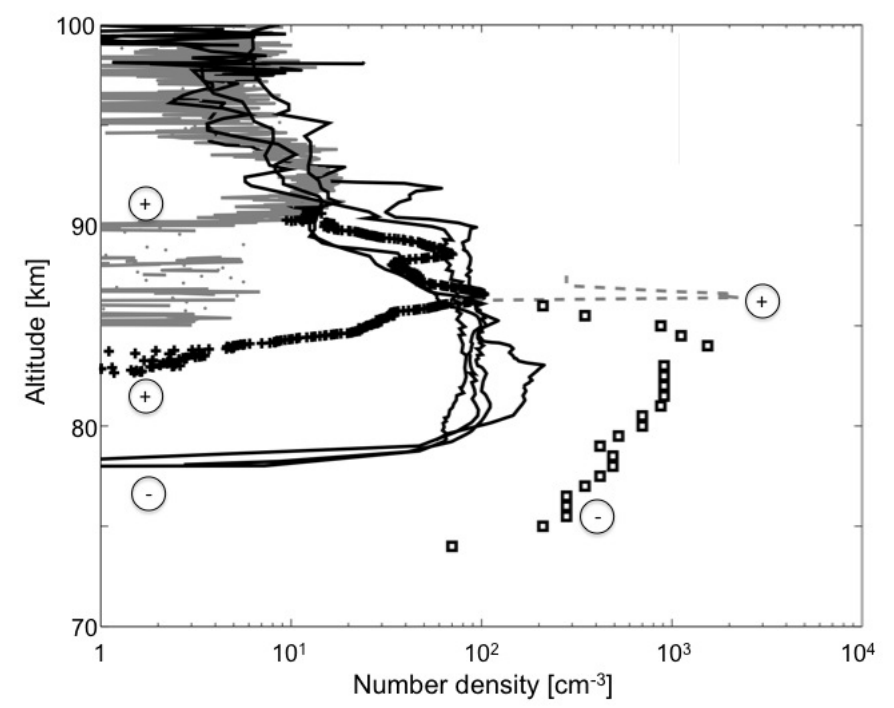

Fig. 5 The reported in-situ measurements of meteoric smoke in the ionosphere. The data are from Gelinas et al. (1998, grey solid line), Horanyi et al. (2000, grey dashed line and squares), Lynch et al. (2005, black solid line), and Rapp et al. (2005, crosses). Grey solid line, grey dashed line and crosses denote measured positive charges and the black solid line and the squares denote detected negative charges. The measurements are further discussed in the text and this figure is adapted from Megner (2008)

Mitchell et al. (1995) and Croskey et al. (2001) sampled both light (2200 amu) and heavy $(20000 \mathrm{amu})$ positively charged particles with concentration $10^{8}-10^{10} \mathrm{~m}^{-3}$ during the rocket flights at altitudes $80--90 \mathrm{~km}\left(3 \cdot 10^{-24}-3 \cdot 10^{-23} \mathrm{~kg}\right.$, i.e. particles $1-6 \mathrm{~nm}$ in size assuming density $10^{3} \mathrm{~kg} / \mathrm{m}^{3}$ ). These observations were performed during the noctilucent cloud season at high latitude, so it remains unclear whether these particles represent meteor smoke or water vapor condensation products. During later flights in Alaska a layer of negatively charged particles with masses 5000-10000 amu and concentration $\sim 10^{8} \mathrm{~m}^{-3}$ $\left(8 \cdot 10^{-24}-2 \cdot 10^{-23} \mathrm{~kg}\right.$ ) were observed (Lynch et al. 2005; Gelinas et al. 2005). The authors suggest that these charged particles represent $10-20 \%$ of the total number of dust particles within this size range (we will further discuss dust detection in Section 6). These latter flights were accompanied by lidar observations of $\mathrm{Na}$ and $\mathrm{Fe}$ atom layers and the observations suggested correlation between charged particle distribution and distribution of the iron atoms. 
3.8 Possible instabilities in the Earth's ionosphere

In the ionosphere, charged dust occurs naturally in the meteor ablation zone $(\sim 80-120$ $\mathrm{km})$ and in particular in polar mesospheric regions such as NLC and PMSE regions ( $80-95 \mathrm{~km}$ altitude). At these altitudes, Debye lengths can range from about centimeters to millimeters, depending on plasma conditions such as daytime or nighttime, as well as factors such as precipitating particle fluxes. Estimates for the number density of small dust particles in the ionosphere, of size $\sim$ a few tens of nm to sub-visual, appears generally to be larger than about $10^{8} \mathrm{~m}^{-3}$ under PMSE conditions (Havnes et al. 2001), so these plasmas may be considered as "dusty plasmas".

Although it has long been thought that dust can form from ablated meteoroid material (Rosinski and Snow, 1961; Hunten et al, 1980), little is known about the sizes or number density of dust in meteor trails. To get an idea of a limit on the dust density in a meteor trail, let us assume that when a meteoroid ablates, all the material condenses and coagulates into dust of size $5 \mathrm{~nm}$. If the meteoroid is initially large, say $10^{-2} \mathrm{~m}$, the average density of dust in a cylindrical trail of size $40 \mathrm{~m}$ by $10 \mathrm{~km}$ would be on the order of $\sim 10^{10} \mathrm{~m}^{-3}$. Since this value could be comparable to the plasma density in the trail, the dust, if charged, might affect the collective behavior of the meteor trail plasma including the behavior of waves and instabilities. Note that charged dust in a meteor trail may affect trail diffusion. It has been suggested that the presence of negatively charged dust may slow down the diffusion by reducing electron diffusivity, similarly to the possible effect of negatively charged dust in preserving plasma irregularities in PMSE regions (Kelley et al. 1998; Zhou and Kelley 1997). Kelley (2004) presented a new explanation for long duration meteor trains involving persistent charged dust trains. It has also been suggested that the effects of background dust on the decay time of underdense meteor trails might provide information on the subvisual smoke particles that are thought to exist (Havnes and Sigernes 2005).

In the upper D -lower E regions of the ionosphere which includes the altitudes of PMSE and the meteor ablation zone where trails form, the electrons have small gyrofrequency compared to their collision frequency (they are magnetized) while the ions are unmagnetized with collision rates larger than their gyrofrequency. In the lower E region, electron $\mathbf{E} \times \mathbf{B}$ or cross-field drifts can drive the known electrojet instabilities, such as the Farley-Buneman instability. These kinds of ion wave instabilities have been observed by radar returns from both equatorial and auroral electrojet regions, as the excited waves satisfy the Bragg scatter condition that the wavelength is $1 / 2$ the radar wavelength (see e.g. Kelley 1989).

It has been suggested that these types of instabilities may apply to the formation of irregularities in meteor trails (Chapin and Kudeki 1994; Pellinen-Wannberg and Wannberg 1996). Oppenheim et al. (2003) and Dyrud et al. (2002) showed that Farley-Buneman type instabilities could occur in meteor trails, driven by electron cross-field drifts due to plasma gradients or ambipolar electric fields in the trail, and that the instabilities can lead to nonspecular meteor trail echoes. Thus, it is of interest to understand how charged dust can affect these and related wave instabilities in the lower ionosphere.

Charged dust may affect the Farley-Buneman instability (Rosenberg and Chow 1998). As discussed above, dust can affect the phase speed of ion waves. At altitudes $<95 \mathrm{~km}$, the phase speed increases as the negative charge density of the dust increases, and in analogy with ion acoustic waves, the critical electron drift decreases so the Farley-Buneman instability could be more easily excited.

The possibility of ion acoustic instability in dusty meteor trails in the low E region was considered by Rosenberg and Merlino (2007). When $T_{e} \sim T_{i}$ (often the case in ionospheric plasmas), the critical electron drift, parallel to $\mathbf{B}$, for an ion acoustic instability is roughly 
$\sim$ the electron thermal speed. However, if the dust charge density is comparable to the ion density $n_{i}$, the ion acoustic speed increases, ion Landau damping decreases, and the critical electron drift decreases, as discussed in section (2.4). Rosenberg and Merlino (2007) considered an expanded trail (radius 10 meters, length $10 \mathrm{~km}$ ) at an altitude $H \sim 95 \mathrm{~km}$, with $n_{i} \sim 10$ times larger than nighttime background plasma density, containing negatively charged dust of size $\sim 1 / 2 \mathrm{~nm}$ with $Z_{d} \sim 1$ and $n_{d} \sim n_{i}$. It was found that the critical electron drift could be reduced by $\sim 1 / 4$ when $n_{i} / n_{e} \sim 10$, implying parallel electric fields on the order of $10-20 \mathrm{mV} / \mathrm{m}$ for ion acoustic wave excitation. Perhaps this mechanism may have some relevance to the observations of enhanced ion acoustic echoes in meteor trails reported by Pellinen-Wannberg and Wannberg (1996) but further work is needed to scope out the range of dust and meteor trail parameters required for instability.

The possibility of a very low frequency dust acoustic instability in a dusty meteor trail in the upper mesosphere was considered by Rosenberg and Shukla (2000; 2002). A relatively large dust charge is favorable for dust acoustic instability, but even larger dust of size tens of nm would get charged negatively to only a few electron charges in the cold ionosphere. Rosenberg and Shukla (2000) suggested that if the dust has low work function $(<4 \mathrm{eV})$, grains could get charged positively to larger charge states by photoemission in daytime conditions (see also Havnes 1990). Assuming positively charged dust with charge density comparable to $n_{i}$, it was shown that a dust acoustic instability might occur in an expanded trail (radius $\sim$ tens of meters), driven by an electron $\mathbf{E} \times \mathbf{B}$ drift of speed less than the ion thermal speed (Rosenberg and Shukla, 2000). The parameters corresponded to a large meteor, with estimated dust mass in the trail $\lesssim 100$ g. Also D'Angelo (2003) discussed a possible cross-field dust acoustic instability in the E region where the ions as well as the electrons are collision dominated.

D'Angelo (2005) considered whether ion acoustic and dust acoustic instabilities driven by electron or ions streaming along a vertical electric field in the high latitude $H \sim 85$ $\mathrm{km}$ ionosphere could be a cause of PMSE. D'Angelo (2005) points out that some observed features of PMSE (such as an anti-correlation of electron fluctuations relative to ion and dust fluctuations) point toward dust acoustic waves as a possible cause of the strong radar returns, but large electric fields $(\sim 1 \mathrm{~V} / \mathrm{m})$ would be required to excite the waves. Observations of large electric fields in these regions, up to $1 \mathrm{~V} / \mathrm{m}$, have been reported (Zadorozhny et al. 1993) although there is controversy regarding this value, with other experiments finding fields $\sim$ a few tens of $\mathrm{mV} / \mathrm{m}$ (e.g. Holzworth et al. 2001). Scales and Ganguli (2004) also considered dust acoustic instability as a possible cause of PMSE, with the instability driven by ion or electron flows in the boundary of localized dusty plasma clouds in the mesosphere.

Dusty plasma instabilities at higher altitudes in the upper $\mathrm{E}$ - lower $\mathrm{F}$ region have been investigated in relation to radar scattering from rocket exhaust plumes. Bernhardt et al. (1995) suggested a dusty plasma mechanism, among others, to explain the enhanced radar backscatter from space shuttle exhaust observed with the Arecibo $430 \mathrm{MHz}$ radar. The mechanism involves the condensation of water vapor exhaust into ice dust particles that get charged, leading to an instability (e.g., dust acoustic instability) due to the streaming of the charged dust relative to the background plasma. This could lead to radar scattering when the excited waves satisfy the Bragg scattering condition. Later, Rosenberg et al. (1999) considered a lower hybrid (LH) instability driven by a beam of negatively charged dust streaming across $\mathbf{B}$, finding that this instability could be relevant for explaining the enhanced radar backscatter from space shuttle exhaust. Rosenberg and Sorasio (2006) have considered the possibility of LH instability in dust-gas formations from rocket exhausts injecting combustion products into the ionosphere (see Platov 2004). 


\section{Dust in the interplanetary medium}

Dust grains in interplanetary space are affected by impacts of the solar wind ions which cause sputtering. Some of the impacting ions become implanted in the dust and can be released as neutrals. Dust-dust collisions lead to fragmentation and vaporization. The vaporized material as well as the released neutrals become ionized and form a subpopulation of the solar wind pick-up ions. The dynamics of the dust is determined by gravity and the Poynting-Robertson force. The Lorentz force, which for the larger grains is a small perturbation, becomes dominant for the small grains, in particular the nano dust, which can be accelerated by the Lorentz force to velocities comparable to the solar wind velocity. Such fast nano dust was recently discovered by the STEREO spacecraft.

After describing dust destruction and dust interactions (section 4.1) we consider the evolution of dust generated species in the solar wind and shortly address the connection of dust to the measured pick-up ions, the neutral solar wind and the interplanetary field enhancements (section 4.2). We then discuss the dust dynamics and elaborate the acceleration of nano dust in the solar wind (4.3).

\subsection{Dust destruction and dust interactions}

A fraction of the impinging solar wind leaves the dust after interaction. The penetration depth $\Delta$ of solar wind protons into dust material is of the order of several $10 \mathrm{~nm}$, the exact depth depending on the dust material. Ions that pass through the dust particles with radius $\mathrm{R}<\Delta$ generally leave the dust in different charge state, often neutral (Minato et al. 2004). The momentum transfer onto the dust leads to the plasma Poynting-Robertson effect (Section 4.3). The solar protons and other solar wind ions impinging on large $(R>>\Delta)$ dust particles remain in the surface layer. Diffusion of the implanted species, recombination and chemical reactions may occur. The outer layers of the dust grains that remained in the interplanetary medium for a couple of hundred years are supposedly saturated with solar wind particles, similar to the lunar regolith (Banks 1971). Fahr et al. (1981) suggested that solar wind particles impinging on such a saturated dust surface recombine and cause the trapped species to be released as neutrals again ("desorption"). Impinging particles also deposit heat, sputter ions out of the dust, damage the lattice structure and modify the mineral composition.

An important process that releases dust material is sputtering. Mukai and Schwehm (1981) considered sputtering of cosmic dust analog materials magnetite (crystalline structure of $\mathrm{Fe}_{3} \mathrm{O}_{4}$ ) and obsidian (glassy material consisting to $2 / 3$ of $\mathrm{SiO}_{2}$ and of $\mathrm{MgO}$ and $\mathrm{Fe}_{3} \mathrm{O}_{4}$ ) by protons and $\alpha$ particles and found that the dust material is predominantly sputtered by impinging protons of energies beyond typical solar wind energy, in majority by solar energetic particles. The sputtering rate is higher for He and hence increases with increasing He abundance. For the dust species and interplanetary medium conditions considered Mukai and Schwehm find that the dust particles suffer only moderate erosion by sputtering before reaching the dust sublimation zone (see Section 5.2.5 related laboratory studies). Dust near the Sun can also be destroyed by rotational bursting (Misconi 1993, Abbas 2004). The dust PR effect varies and is enhanced during coronal mass ejections (Ragot and Kahler 2003).

The dust and meteoroids are subject to collisional fragmentation, which may account for their size distribution in the interplanetary medium (Dohnanyi 1978). The dust collisional fragmentation and vaporization processes were discussed by Tielens et al. (1994) and Jones et al. (1996) . The impact of the dust particle generates a shock wave in the target grain. The 
evolution of the shock wave within the particles can be studied in analogy with the corresponding wave in the bulk solid, with the experiments, although mostly restricted to lower collision velocities $(<\mathrm{km} / \mathrm{s})$ and larger projectile sizes $(\mathrm{mm}$ and $\mathrm{cm})$ providing additional information. In this way the authors obtained some results concerning the distribution of fragments and the vaporized mass which were applied to dust collisions in the interstellar medium. Vapour production requires high collision velocities $(>10 \mathrm{~km} / \mathrm{s})$ and in the interplanetary medium it usually occurs in the inner solar system (Mann and Czechowski 2005).

Neutral species are quickly ionized in the interplanetary medium (see Section 4.2), but a fraction of the forming vapor is already ionized. Calculations by Hornung with a hydrodynamics code predict that for typical impact velocities the vapour is in neutral charge state and that singly or doubly ionized species only form for impact velocities of the order of $100 \mathrm{~km} / \mathrm{s}$ (Hornung et al. 2000). Charge production is, however, already observed for lower speeds, of the order of few $\mathrm{km} / \mathrm{s}$. Laboratory experiments at single charged grains accelerated in an electrostatic accelerator show that the charge released upon impact increases with the impact speed roughly as

$$
Q \simeq 0.7 m^{1.02} v^{3.48}
$$

where charge $Q$ is in Coulombs, mass $m$ is in $\mathrm{kg}$ and impact speed $v$ is in $\mathrm{km} / \mathrm{s}$. There is a deviation from this relation for small velocities, where charge production is higher (McBride and McDonnell 1999).

Dust particles sublimate when they reach vaporization temperature. Describing dust sublimation in space requires extrapolation from the results obtained under laboratory conditions. Though the conditions vary with size and composition, sublimation becomes important within $0.1 \mathrm{AU}$ (about 20 solar radii) from the Sun. The sublimation distances cannot be derived from observations. A canonical value of a dust free zone radius around the Sun is about 4 solar radii where most dust compounds sublimate, even though calculations show that some dust particles may survive further inward (see Mann et al. 2004 for a review).

According to a dust fragmentation model (Mann and Czechowski 2005) the majority of ions that are generated from the dust in the region outside roughly $0.1 \mathrm{AU}$ from the Sun are produced during dust collisions. The production rates of pick up ions between 0.1 and $1 \mathrm{AU}$ are $(0.14-1.2) \cdot 10^{3} \mathrm{~kg} \mathrm{~m}^{-3}$ from solar wind desorption, $<0.14 \cdot 10^{3} \mathrm{~kg} \mathrm{~m}^{-3}$ from solar wind passing dust and $(0.17-36) \cdot 10^{3} \mathrm{~kg} \mathrm{~m}^{-3}$ from collisional vaporization (Mann and Czechowski 2005). The pick up ions production from sungrazing comets is at average $0.16 \cdot 10^{3} \mathrm{~kg} \mathrm{~m}^{-3}$ and its flux near Earth peaks between August and January (Bzowski and Krolikowska, 2005).

\subsection{Dust generated species in the solar wind}

Once the molecular and atomic species are released they are ionized; the major ionization mechanisms are photo ionization, charge exchange ionization with solar wind protons, and electron impact ionization close to the Sun (Mann and Czechowski 2005). The neutrals released inside of $1 \mathrm{AU}$ are ionized on time scales small compared to the dust orbital periods. The ionized atoms or molecules are then subjected to Lorentz forces and gyrate about the magnetic field lines transported with the solar wind. This was studied in detail by Vasyliunas and Siscoe (1976). The ions are picked up by the solar wind within the Larmor rotation time of the order of seconds. (The Larmor rotation time for a proton at $1 \mathrm{AU}$ is $300 \mathrm{~s}$ and inversely proportional to $\mathrm{q} / \mathrm{m}$ ). The ion initial velocity distribution ranges from zero to twice the solar 
wind speed in the resting frame. It evolves as result of pitch angle scattering within the solar wind. Measuring the velocity distribution of pick-up ions allows to infer the distance from the Sun, where the ions were generated. The interstellar gas that enters the solar system provides the major component of neutrals in the interplanetary medium and also the major source of pick up ions.

If released as neutrals, then the dust-generated ions that are carried in the solar wind are predominantly singly charged, except for those that are released very close to the Sun. Species that are generated by dust destruction in the inner corona where the charge states of solar wind ions are determined by electron collisions may not be distinguished from the solar wind. Lemaire (1990) hence considered the infall of heavy ions from dust into the solar corona and its possible influence on the solar wind composition.

A part of the Solar Wind Ion Composition Spectrometer (SWICS) experiment aboard the Ulysses spacecraft comprises a detailed study of the pick-up ions in order to quantify some interstellar neutral gas components that enter the solar system (see Gloeckler et al. 2010 and references therein). Aside from pick-up ions that are generated by interstellar neutrals, SWICS discovered the pick-up ions associated with a so-called "inner source", which originates from the vicinity of the Sun and includes the elements $\mathrm{H}, \mathrm{He}, \mathrm{C}, \mathrm{N}, \mathrm{O}$, $\mathrm{Mg}, \mathrm{Si}, \mathrm{Ne}$, and molecular ions (see Gloeckler et al. 2010 and references therein). Attempts to explain the inner source with the dust surface scattering process suggested by Fahr et al. (1981) failed to explain the amount of detected ions (Mann et al. 2010a).

Dust related sources were also discussed in the context of neutral measurements, again without quantitative agreement (ibid.) A neutral atom released from dust, if ionized by charge exchange with the solar wind ion, gives rise at the same time to a pick up ion and a neutral solar wind atom. In consequence the flux of neutral solar wind hydrogen generated in this way should be similar to the total flux of inner source pick ions generated from dust. Collier et al. (2003) considered a similar mechanism, but assumed that the charge exchange occurs on the dust surface. He found that to account for the observed neutral solar wind flux a very large value of dust geometric cross section integrated between the Sun and $1 \mathrm{AU}$ is necessary.

The mass loading by species released in cometary dust trails was also considered in the context of Interplanetary Field Enhancements (IFEs). IFEs are observed features in the time profile of the interplanetary magnetic field that seem neither instrumental nor correlated to other solar wind properties. Experimenters suggested their association to cometary dust trails or to other dust sources (Jones et al. 2003, Russell 2010). Mann et al.( 2010a) estimated the charge production rate due to collisions within the dust trail and due to collisions of trail particles with interstellar dust and found that, with the current empirical models of vapour production, the collision rates are far too small to generate amounts of gas comparable to the solar wind densities.

\subsection{Dust dynamics in the interplanetary medium}

The main forces acting on dust in the interplanetary medium are solar gravity $\mathbf{F}_{\mathrm{g}}$, radiation pressure $\mathbf{F}_{\text {rad }}$, solar wind pressure and the Lorentz force.

The radiation pressure force in the particle rest frame can be separated into the direct radiation pressure force and the velocity-dependent term that is referred to as the PoyntingRobertson (P-R) drag force. The latter causes the gradual loss of orbital angular momentum and orbital energy. The dust migrates toward the Sun. The momentum transfer from impacting solar wind particles, similarly to the radiation pressure force, can be decomposed into 
direct solar wind pressure and the solar wind drag. Even though the force due to the plasma drag is much smaller in magnitude than the radiation pressure force, the corresponding drag on the orbital motion is comparable to the PR drag (plasma or pseudo Poynting-Robertson (PR) effect, e.g. Mukai and Yamamoto 1982; Minato et al. 2004). This results from the greater aberration angle than in the case of photon impact, since the solar wind speed is much smaller than the velocity of light. The PR lifetimes of dust within 1 AU are smaller than $10^{6}$ years. The plasma PR effect varies with the solar cycle. It may have been more significant in the early solar system, which presumably had a stronger solar wind (Minato et al. 2006). Newly formed particles for which $\mathbf{F}_{\mathrm{g}} \sim \mathbf{F}_{\text {rad }}$ can be ejected outward in hyperbolic orbits. The exact conditions depend on the dust initial velocity and on the value of the radiation pressure force. This is typically the case for dust in the size interval of a few 0.1 to several $\mu \mathrm{m}$ and these particles are called $\beta$-meteoroids (see Fig. 2).

Let's now consider the Lorentz force, the relevant parameter for which is the charge to mass ratio $\mathrm{q} / \mathrm{m}$, which is proportional to $1 / R^{2}$ ( $\mathrm{R}$ is the grain radius, deviations from this proportionality are discussed in Section 5). Assuming surface potential of $\sim 5 \mathrm{~V}$, the charge to mass ratio for a dust particle with $\sim 1 \mu \mathrm{m}$ radius is $\sim 510^{-10} \mathrm{e} / \mathrm{m}_{p}$ (with elementary charge, e and proton mass, $\mathrm{m}_{p}$ ). The corresponding Larmor frequency $\Omega_{L}$ at $1 \mathrm{AU}$ can be found from the relation $\Omega_{L} \approx(q / m) 10^{4} \cdot \mathrm{B} \mathrm{s}^{-1}$ where $\mathrm{q} / \mathrm{m}$ is in $\mathrm{e} / \mathrm{m}_{p}$ and the magnetic field $B$ in Gauss. Taking $B \sim 30 \mu \mathrm{G}$ gives $\Omega_{L}=1.5 \cdot 10^{-10} \mathrm{~s}^{-1}$ which is much less than the orbital frequency at $1 \mathrm{AU}$. During orbital motion, such dust particles successively pass the regions of different field directions pushing them away from the initial orbital plane. Hence, the main effect of the Lorentz force on the $\sim 1 \mu \mathrm{m}$ dust particles is the spread of the orbits in inclination caused by the sector structure of the interplanetary magnetic field (Morfill and Grün 1979). Lorentz force becomes more important for smaller particles. The dynamics studies of the dust with sizes of the order $1 / 10 \mu \mathrm{m}$ are reviewed by Mann et al.(2004) and further references are given there.

The following discussion of the fluxes of smaller nano dust is based on a paper by Czechowski and Mann (2010). To study the trajectories of nano dust in the solar magnetic field we use Parker's model (Parker 1958)

$$
B_{r}= \pm B_{0}\left(\frac{r}{r_{0}}\right)^{-2} \quad B_{\phi}= \pm B_{0}\left(\frac{r}{r_{0}}\right)^{-1} \cos \theta \quad B_{\theta} \equiv 0
$$

where $\mathrm{r}_{0}=1 \mathrm{AU}$, and $B_{0} \simeq 2-4 \times 10^{-9} \mathrm{~T}(20-40 \mu \mathrm{G})$. Except for the immediate vicinity of the Sun or the regions directly above the solar poles, this model provides a good approximate description of the average magnetic field. Near solar minimum, the fields in the northern and in the southern hemispheres correspond to opposite polarity. The dividing line is tilted relative to the ecliptic plane: this leads to the sector structure of the interplanetary field. The sectors with opposite polarity are separated by a thin current sheet which is warped due to the effect of the tilt. The thickness of the current sheet is of order of $10^{5} \mathrm{~km}$. Near solar maximum the field configuration is more complex. However, the simple picture of a tilted dividing line was found useful even in this case, although with a high $\sim 90$ deg tilt.

We assume that the charge to mass ratio of a dust particle of $\sim 10 \mathrm{~nm}$ radius is approximately $q / m=10^{-5} e / m_{p}$. At the distance $\mathrm{r}=0.1 \mathrm{AU}$ from the Sun the solar magnetic field $B \simeq 3 \times 10^{-7} \mathrm{~T}$ gives for such a dust particle the Larmor frequency $\Omega_{L}=3 \times 10^{-4} \mathrm{~s}^{-1}$. The Larmor radius $\mathrm{R}_{L}$ is equal to $\left|v_{\perp}^{\prime}\right| / \Omega_{L}$ where $v_{\perp}^{\prime}$ is the velocity perpendicular to the magnetic field in the frame where the electric field $\mathbf{E}=0$. In relation to the inertial frame centred at the Sun, this frame moves at the velocity equal to the transverse component of the solar wind velocity with respect to $\mathbf{B}$, corresponding to approximately $45 \mathrm{~km} / \mathrm{s}$ at $r=0.1$ 


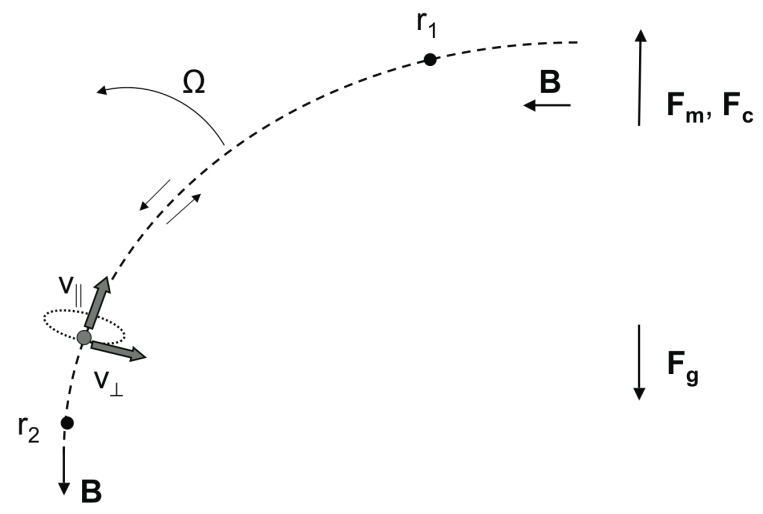

Fig. 6 Sketch of the nano dust trajectory in guiding center approximation. The freshly created dust particle is governed by solar gravity $\mathbf{F}_{\mathbf{g}}$ directed inward, and centrifugal force $\mathbf{F}_{\mathbf{c}}$ and mirror force $\mathbf{F}_{\mathbf{m}}$, directed outward. It gyrates about the magnetic field line (dashed line) with the velocity $\mathbf{V}_{\perp}$ and follows magnetic field line rotation. A trapped particle oscillates along it. Particles are ejected when the combination of centrifugal and magnetic mirror force exceeds gravity.

AU. Assume that the dust particle is a fragment split off the larger dust particle moving at Keplerian velocity. The Larmor radius can then be seen to be $R_{L}=1.5 \cdot 10^{8} \mathrm{~m}$. The condition $R_{L}<<r$ (and $R_{L}<<L$ where $\mathrm{L}$ is the characteristic scale of the field) is therefore satisfied with a large margin. By similar reasoning, for $r=0.3 \mathrm{AU}$ one obtains $R_{L}=3 \cdot 10^{9} \mathrm{~m}$, so that $R_{L}<<r$ still holds. It follows that in a sizeable part of the inner $(r<1 \mathrm{AU})$ heliosphere the adiabatic condition $R_{L}<<L$ is satisfied for $\sim 10 \mathrm{~nm}$ (or smaller) dust particles. The guiding centre approximation (e.g. Northrop 1963) provides then a good description of the dynamics.

The guiding centre velocity in the direction perpendicular to the field line is approximately equal to the component of the solar wind velocity that is transverse to $\mathbf{B}$. In effect, the guiding centre motion consists approximately of sliding along the rotating magnetic field line. In Czechowski and Mann (2010) we found that the motion of the nano dust particle following from exact integration of the dust equation of motion can be understood qualitatively within the simple picture following from the guiding centre approximation. The rotation of the field line induces inertial forces, in particular the centrifugal force, acting in the direction opposite to that of the gravity force. The inertial force and the "magnetic mirror" force are independent on the value of $q / m$, implying that, in the region in which the guiding centre approximation works $\left(R_{L}<<L\right)$, the dynamics becomes approximately independent on the dust particle size.

Near the ecliptic, the centrifugal force overcomes gravity at about $r=0.15$ AU. It can be seen that only the dust particles released at distances larger than this critical value do escape from the vicinity of the Sun and can reach $1 \mathrm{AU}$. The motion of the dust particles released at smaller distances can be understood as a bounded motion along the rotating field line between the initial (release) distance and the perihelium distance, where the particle is reflected by magnetic mirror. 


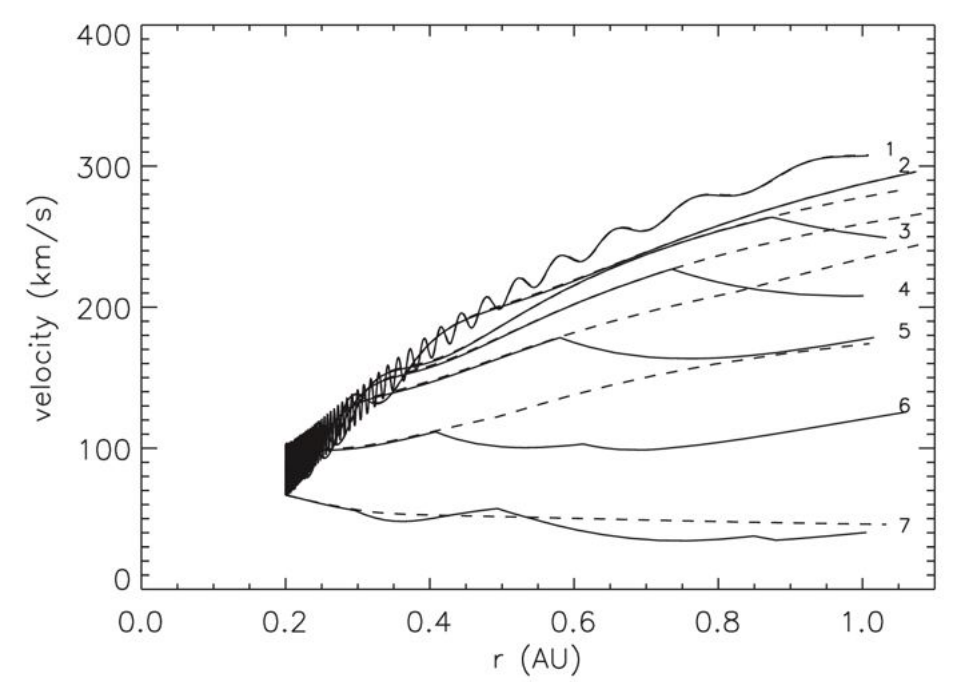

Fig. 7 The speed vs. distance for nano dust released at $0.2 \mathrm{AU}$ (solid and dashed lines) from top to bottom with $q / m=10^{-4}, 10^{-5}, 7 \cdot 10^{-5}, 5 \cdot 10^{-5}, 3 \cdot 10^{-5}$, and $10^{-5} e / m_{p}$ (from Czechowski and Mann 2010).

At $1 \mathrm{AU}$, a significant part of the $\sim 10 \mathrm{~nm}$ or smaller dust particles would come from the region near the Sun, where the collisional fragmentation rate of larger dust particles is high. Although at the distances approaching $1 \mathrm{AU}$ the guiding centre picture ceases to be applicable to $10 \mathrm{~nm}$ size grains, the particles are are still approximately tied to rotating magnetic field lines. In result, their velocity at $1 \mathrm{AU}$ is of the order of the velocity of the field line, which is of the order of the solar wind velocity. At the same time, these dust grains have an outward velocity component, acquired at smaller distances due to the centrifugal force (see Fig. 6). Fig. 7 shows the absolute velocity as function of distance for nano dust with different $\mathrm{q} / \mathrm{m}$ released at $0.2 \mathrm{AU}$. The solid lines denote trajectories during focusing field configuration with incoming magnetic field in the northern hemisphere, so that the electric field $-(1 / \mathrm{c}) \mathrm{V} \times \mathrm{B}$ points toward the current sheet. The dashed lines correspond to reversed antifocusing field configuration with outgoing magnetic field in the northern hemisphere and electric field pointing away from the current sheet. Trajectories with $q / \mathrm{m}=10^{-4} \mathrm{e} / \mathrm{m}_{p}$ and $q / m=10^{-5} e / m_{p}$ are almost identical for the two field configurations and reach highest velocity. Acceleration quickly drops for smaller $\mathrm{q} / \mathrm{m}$ (larger mass). Crossing the current sheet (sharp bend in the velocity profile) also decreases the effect of acceleration. Grains with $q / m=10^{-7}$ (about $100 \mathrm{~nm}$ ) are ejected with moderate speed. Dust with sizes $\sim 10 \mathrm{~nm}$ or smaller near $1 \mathrm{AU}$ should therefore in majority stream outward with velocity $\sim v_{S W}$ ). The trajectory calculations combined with a dust collision model lead to an estimated flux of dust with mass $\mathrm{m}>10^{-20} \mathrm{~kg}$ of $\phi=0.6 \mathrm{~m}^{-2} \mathrm{~s}^{-1}$ (Czechowski and Mann 2010).

Fast nano dust particles have recently been detected by the wave instrument of the STEREO spacecraft. The measured flux is highly time variable (Meyer-Vernet et al. 2009b). The estimated average flux of nanoparticles detected by STEREO during the year 2007 is shown in Fig. 2. It is of the same order as the estimate based on trajectory calculations and the collision models (Czechowski and Mann 2010).

Both the nano dust and the larger $\beta$-meteoroids cross 1 AU in outbound orbits, but have distinctly different velocities. Fig. 8 shows the average absolute velocities as func- 
tion of grain mass. Though the Ulysses dust instrument made most of its measurements beyond $1 \mathrm{AU}$ we compare the calculated velocities with the Ulysses results. The detected $\beta$-meteoroids have velocities $\sim 40-80 \mathrm{~km} / \mathrm{s}$ (Wehry and Mann, 1999), comparable to the shown values for the dirty silicate asteroidal dust model. The Ulysses measurements cover masses $>10^{-19} \mathrm{~kg}$ which is larger than the nano dust.

The trajectory calculations do not provide final explanation of the flux variations. The nano dust presumably forms by fragmentation of dust of several micrometer sizes and larger, and we are unable to explain the high variability of the flux with variations of the dust source. The variations in the extension of the trapping zone would lead to time variation of the flux near $1 \mathrm{AU}$, but possibly also other mechanisms come into play. Since the nano dust is accelerated continuously along its trajectory, it is likely that the time variations are connected to solar wind conditions in the inner solar system, rather than to conditions near the spacecraft. We note that further analysis of the STEREO/WAVES measurements shows, aside from the detected nano dust, a second class of dust impacts that are possibly due to $\beta$-meteoroids and interstellar dust (Zaslavsky and Meyer-Vernet, work in progress).

Because of its high rigidity we can not exclude that the nano dust enters the Earths magnetosphere. If this is the case, the mass input into the ionosphere below $400 \mathrm{~km}$ altitude is $\sim 2 \cdot 10^{-6} \mathrm{~kg} \mathrm{~s} s^{-1}$ or $55 \mathrm{~kg} /$ year, which is $\sim 10^{-6}$ or less of the meteoroid mass input. Nano dust impacts are also suspected to generate impact craters detected on a foil that was exposed to space within the Earth magnetosphere on the International Space Station ISS (Carpenter et al. 2005) (also shown in Fig. 2). The possible connection of the flux derived from the impact craters to the flux derived from STEREO results was not studied.

In spite of the large speed of the nano dust, the total momentum carried by the nano dust component is small. Based on the Czechowski and Mann (2010) flux estimate the average momentum of nano dust at $1 \mathrm{AU}$ is $6 \cdot 10^{-21} \mathrm{~kg} \mathrm{~m} \mathrm{~m}^{-2} \mathrm{~s}^{-1}$ and this is $\lesssim 10^{-6}$ of the solar wind momentum assuming solar wind mass density from table $2.4 \_2$ and solar wind speed $400 \mathrm{~km}$ / $\mathrm{s}$. The trajectory calculations described above assume fixed values of $\mathrm{q} / \mathrm{m}$. Comparison of the $\mathrm{q} / \mathrm{m}$ values used in our calculation with the values obtained in dust charging calculations by Mukai (1981) and Kimura and Mann (1998) can be found in the figure 11 of Czechowski and Mann (2010). The inverse square size $1 / R^{2}$ scaling of the charge to mass ratio changes in the small size limit, because secondary electron emission becomes more important near the Sun and moreover small particle effects arise for the charging processes. This is further discussed in Section 5.

\section{Dust charging and laboratory experiments related to dust in space}

In the interplanetary medium and in the ionosphere the dust charging is based on the same physical processes, though the relevant parameters cover quite different ranges. Laboratory studies concerned with dust charging and other interaction of dust with electrons, ions and photons require experiments with single dust grains. This is accomplished in several laboratories by using quadrupol traps. For most of the experiments high vacuum conditions are required. Experimental results mainly confirm theory, but show the importance of effects due to the dust size, dust shape, as well as surface reactions and in general deviation from theory for small sizes. The measurements of secondary electron emission (SEE) reveal a component of low energy backscattered electrons, which is not predicted by theory. The sputtering experiments suggest that sputtering from heavy solar wind ions impacting on dust is more important for sputtering rates than previously assumed. 


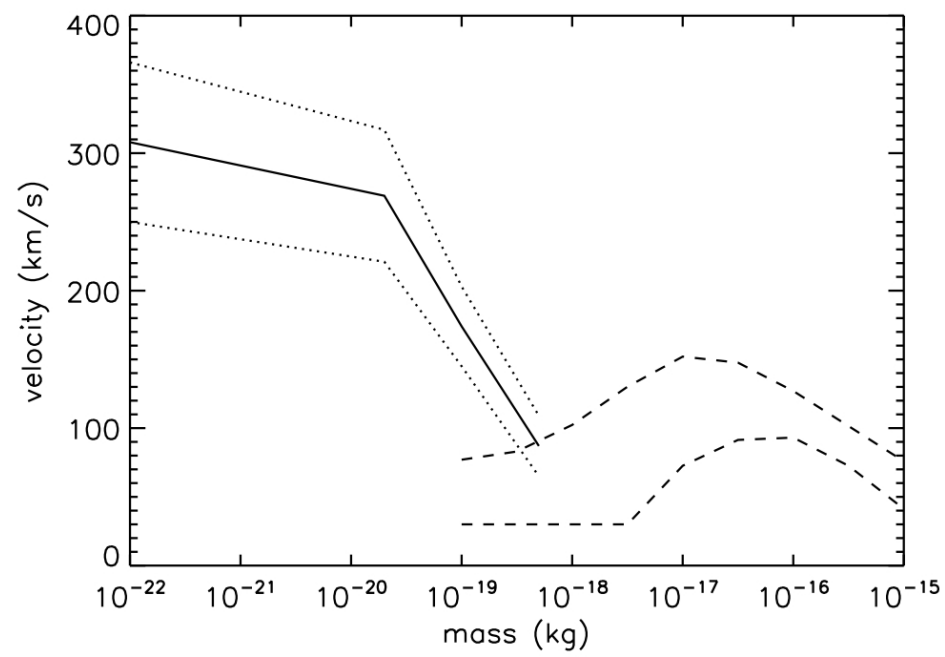

Fig. 8 The average velocity as function of mass at $1 \mathrm{AU}$ for dust ejected by Lorentz forces (solid line and dotted lines) and dust ejected by radiation pressure (dashed lines). The shown solid and dotted lines are derived based on a model of nano dust production by collisional fragmentation inside $1 \mathrm{AU}$ combined with trajectory calculations. The dashed lines show velocities are derived assuming that the dust is released from circular orbit at $0.1 \mathrm{AU}$ and ejected outward by radiation pressure obtained for a highly absorbing cometary dust model (upper curve) and a dirty silicate asteroidal dust model (lower curve). This figure is adapted from Mann et al. (2010b).

This section is organized in a part describing the theory of dust charging (5.1) and in a part describing laboratory experiments to measure specific processes and the related numerical models (5.2).

\subsection{Theoretical description of dust charging}

A dust particle immersed in a plasma and a radiative environment collects charged plasma particles and may emit electrons and ions. In general, the incoming and outgoing electric currents do not balance each other initially, making the dust particle's electrostatic charge (and potential) vary, which in turn changes the currents. This proceeds until the dust particle's charge and potential set up so as to make the currents balance each other (see for example Whipple 1981, Mukai 1981, Goertz 1989, Mendis and Rosenberg 1994).

In the following discussions on dust charging, we consider first dust particles of size larger than the nm scale and approximate them as macroscopic spheres of radius $R_{d}$. We also assume that their separation is greater than the Debye length, that the velocity distributions of the plasma particles are Maxwellian, and only consider the collection of plasma particles and photoelectron emission. We describe the basics of dust charging in the solar wind and in the ionosphere (5.1.1) and then give the discuss the charging currents and electrostatic potentials in these cases (5.1.2) in order to finally describe the electric charge and charging time scale (5.1.3). More complex cases are considered at the end of this section (5.1.4). 


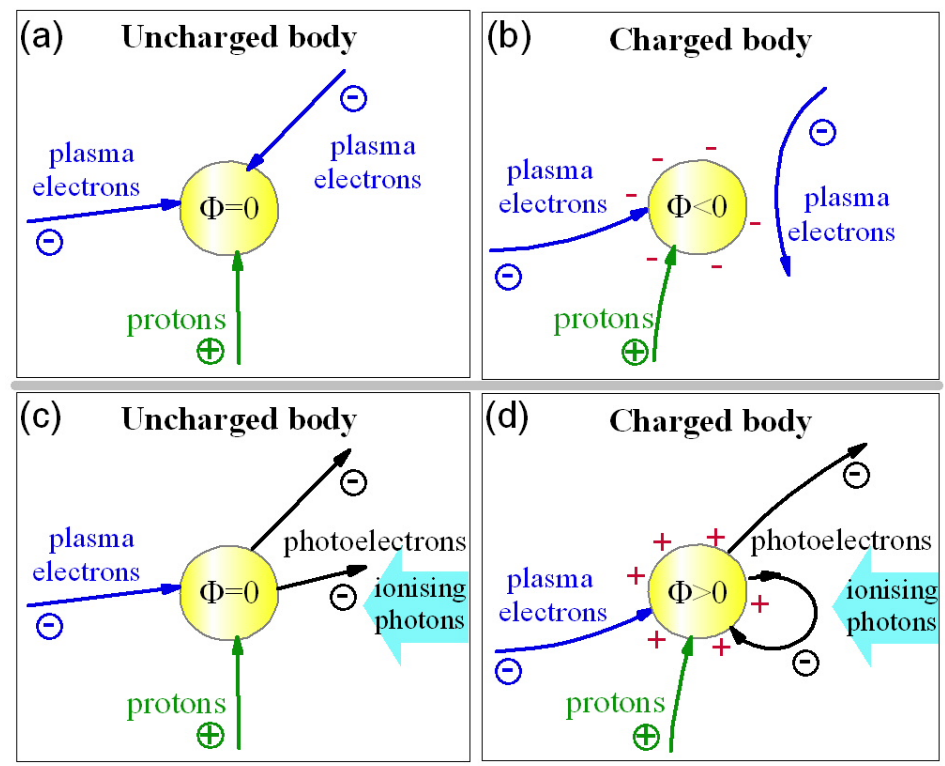

Fig. 9 Sketch of the charging of a body in a plasma (adapted from Meyer-Vernet 2007 page 353). In the ionosphere (top), the charging is governed by collection of plasma electrons and ions, with the former dominating because of their larger mobility (a), so that the dust particle is charged negatively until the electrostatic potential repels enough electrons to make the net current vanish (b). In the interplanetary medium (bottom), the flux of photoelectrons from an uncharged dust particle far outweighs the flux of incoming plasma electrons - itself much larger than the flux of plasma ions (c). This charges the body positively, until the electrostatic potential traps enough photoelectrons to make the net current vanish (d).

\subsubsection{Some basics of dust charging}

We begin the discussion by making order of magnitude estimates (Fig. 9).

Consider first the ionosphere. Photoelectron emission from the dust particle is generally small compared to collection of plasma particles, so that the charge is determined by the ambient electron and ion fluxes. Because of the very small electron mass, the electron flux is initially much greater than the ion flux, so that the dust particle charges negatively, until the negative charge repels sufficiently the incoming electrons to make their flux balance the ion flux. For doing so, the dust particle's electrostatic potential must ensure that the (positive) potential energy $-e \Phi$ outweighs the typical kinetic energy $k_{B} T_{e} \sim 0.03-0.2 \mathrm{eV}$ (depending of the altitude) of the plasma electrons. We deduce that dust particles charge to a negative potential of up to several tenths of volt in the ionosphere.

On the other hand in the interplanetary medium, an uncharged dust particle subjected to the solar ionising radiation generally ejects many more electrons than it collects from the ambient plasma, whereas the incoming ion flux is still smaller. Hence it charges positively, until this positive charge binds sufficiently the photoelectrons to make their flux balance that of the solar wind electrons. For doing so, the dust particle's potential $\Phi$ must provide the photoelectrons with a (negative) potential energy $-e \Phi$ that outweighs their typical kinetic 
energy of a few $\mathrm{eV}$. We deduce that a sunlit dust particle charges to a positive potential of several volts in the solar wind.

\subsubsection{Charging currents and electrostatic potential}

For dust particles much smaller than the Debye length and the collisional free path, the plasma currents are orbit-motion-limited (OML, see Laframboise and Parker 1973). Hence the electron and ion currents on a dust particle of surface $4 \pi R_{d}^{2}$ at (negative) potential $\Phi$ in the ionosphere are

$$
\begin{array}{rlr}
I_{e} & =\pi R_{d}^{2} n_{e} e v_{e} \exp \left(e \Phi / k_{B} T_{e}\right) & \Phi<0 \\
I_{i} & =\pi R_{d}^{2} n_{i} Z e v_{i}\left(1-Z e \Phi / k_{B} T_{i}\right) & \Phi<0
\end{array}
$$

since they are respectively repelled and attracted. Here $Z e$ is the ion charge, $n_{e, i}$ and $v_{e, i}=$ $\left(8 k_{B} T_{e, i} / \pi m_{e, i}\right)^{1 / 2}$ are respectively the electron, ion number density and average speed, so that for a given infinitesimal surface element, $n_{e} / 2$ electrons per unit volume are approaching from one side with an average perpendicular velocity $v_{e} / 2$. The (negative) equilibrium potential $\Phi$ is obtained by balancing the currents as

$$
Z e \Phi / k_{B} T=-\ln \left[\left(m_{i} / m_{e}\right)^{1 / 2}\left(T_{e} / T_{i}\right)^{1 / 2} /\left(1-e \Phi / k_{B} T_{i}\right)\right] \quad \text { ionosphere }
$$

For example, with one singly charged ion species of mass $m_{i} \simeq 30 m_{p}$ and temperature $T_{i} \simeq$ $T_{e} \simeq T$, the dust particle potential is $\Phi \simeq-3.9 k_{B} T / e$ volts.

In contrast, in the interplanetary medium, the dominating current is generally the photoemission current due to solar ionizing radiation. For most materials, the yield, i.e. the number of ejected photoelectrons per absorbed photon, becomes significant for photon energies above several $\mathrm{eV}$, with a maximum yield in the range 0.05-0.5, depending on the physical and chemical structure of the dust particle. The photoemission current from an uncharged dust particle is found by integrating over the solar spectrum the product of the yield by the absorbed flux. It is in the range (Grard 1973; Mukai 1981)

$$
I_{p h 0} \simeq \delta \cdot 10^{14} e R_{d}^{2} / r_{\mathrm{AU}}^{2} \quad \delta \sim 0.6-6
$$

where $r_{\mathrm{AU}}$ is the heliospheric distance in astronomic units and the smaller value of $\delta$ corresponds to low-yield materials such as graphite, the larger to high-yield photoemitters such as metals and silicates. Photoelectrons are ejected with a typical energy of 1-3 eV, which may be approximated by a temperature

$$
T_{p h} \sim 1-2 \times 10^{4} \mathrm{~K}
$$

This holds for an uncharged surface. For a charged surface whose potential $\Phi$ with respect to the distant undisturbed plasma is positive, the escaping photoelectrons are those emitted with a kinetic energy greater than $e \Phi$. Integrating over the velocities, this yields

$$
I_{p h}=I_{p h 0}\left(1+e \Phi / k_{B} T_{p h}\right) \exp \left(-e \Phi / k_{B} T_{p h}\right) \quad \Phi>0
$$

The dust particle potential settles so that this current is balanced by the current of incoming plasma electrons (attracted)

$$
I_{e}=\pi R_{d}^{2} n_{e} e v_{e}\left(1+e \Phi / k_{B} T_{e}\right) \quad \Phi>0
$$


We have neglected for simplicity the solar wind proton contribution, which is smaller than the electron one. The dust particle equilibrium potential is thus given by

$$
\begin{aligned}
\Phi & \simeq\left(k_{B} T_{p h} / e\right) \ln \left[\frac{5 \cdot 10^{9} \delta}{n_{\mathrm{m}^{-3}} T_{e}^{1 / 2} r_{\mathrm{AU}}^{2}} \frac{1+e \Phi / k_{B} T_{p h}}{1+e \Phi / k_{B} T_{e}}\right] \\
& \sim 1-10 \text { volts interplanetary medium }
\end{aligned}
$$

where we have substituted typical solar wind parameters, and neglected the weak radial variation of $T_{e}^{1 / 2}$.

\subsubsection{Electric charge and charging time scale}

The electrostatic charge $q$ on a dust particle of radius $R_{d} \ll L_{D}$ is thus

$$
q \simeq 4 \pi \varepsilon_{0} R_{d} \Phi
$$

With the typical potentials found above, this corresponds to a number of elementary charges

$$
\begin{aligned}
& q / e \sim \text { a few } 10^{8} \times R_{d} \quad \text { (ionosphere) } \\
& q / e \sim \text { a few } 10^{9} \times R_{d} \quad \text { (interplanetary medium). }
\end{aligned}
$$

This amounts to values up to $\sim$ tens and hundreds electrons for a dust particle of size 0.1 $\mu \mathrm{m}$ in respectively the ionosphere and the interplanetary medium.

The above estimates are equilibrium values. The equilibrium time may be estimated by viewing the dust particle in the plasma as an electric circuit of capacitance $C \simeq 4 \pi \varepsilon_{0} R_{d}$ and resistance $R \sim 1 /|d I / d \Phi|$, where $I$ is the current flowing between the dust particle and the plasma in absence of equilibrium, so that $\tau \sim R C$. For each charging process contributing to a current $I$, the derivative is of the order of magnitude of $e I / k_{B} T$, where $I$ and $T$ are respectively the rate and temperature of the charging process that yields the greatest derivative. For example for a dust particle in the solar wind, the charging time scale is of order of magnitude

$$
\begin{aligned}
\tau & \sim C k_{B} T_{p h} /\left(e I_{p h 0}\right) \\
& \sim \frac{5}{R_{d(\mu \mathrm{m})} r_{\mathrm{AU}}^{2}} \mathrm{~s} \quad \text { (interplanetary medium) }
\end{aligned}
$$

for a dust particle of radius $R_{d(\mu \mathrm{m})}$ at distance $r_{\mathrm{AU}}$ from the Sun. This yields a few tens seconds for a $0.1 \mathrm{~m}$ grain at $1 \mathrm{AU}$. Charging time scales are possibly different for the nanometric dust . Laboratory measurements indicate the importance of electron attachment in the charging of nano dust (see below) and this may be relevant to the charging of the meteoric smoke particles that are thought to exist in the mesopause (see Megner et al. 2006).

Let's consider charging of sub-nm dust that is thought to be generated in meteor trails. For example, to estimate if there is enough time for sub-nm dust particle to charge in a meteor trail, we use the time scale for dust charging by attachment of a single electron:

$$
\tau_{e} \sim\left(\pi R_{d}^{2} n_{e} v_{e}\right)^{-1}
$$

(we neglect other charging mechanisms in this estimate). The electron density in the trail at time $t$ can be obtained by dividing the line density of the meteor trail, $\alpha$, by $\pi r^{2}$, where $r$ is the radius of the meteor trail given by

$$
r^{2}=4 D t+r_{0}^{2} .
$$


Here $r_{0}$ is the initial radius that is described in section 3.2 and $D$ is the ambipolar diffusion coefficient; D is about $1 \mathrm{~m}^{2} \mathrm{~s}^{-1}$ at $85 \mathrm{~km}$ and $140 \mathrm{~m}^{2} \mathrm{~s}^{-1}$ at $115 \mathrm{~km}$. This equation applies to the usual meteor ablation zone (see e.g. McKinley 1961). Thus the charging time (for larger t) goes as

$$
\tau_{e} \sim 4 D t /\left(\alpha R_{d}^{2} v_{e}\right),
$$

so $\tau_{e}$ is smaller for larger meteors at lower altitude. For example, consider a meteor of mass $\sim 0.1 \mathrm{~g}$ and line density $\sim 10^{16} / \mathrm{m}$ that ablates and forms a meteor trail at an altitude of about $95 \mathrm{~km}$, where $D \sim 10 \mathrm{~m}^{2} / \mathrm{s}$. For a dust particle of radius $\mathrm{R}$, and for $t=5 \mathrm{~s}$, we find that $\tau_{e} \sim 0.5 \mathrm{~s}$, so it appears the dust particle could charge quickly.

\subsubsection{Additional processes}

The above estimates neglect some processes which may be important in particular cases.

Firstly, electrons that are energetic enough can penetrate into the dust particle and excite electrons within it, producing secondary emission of electrons. This effect may be significant in the interplanetary medium for some materials. In that case it makes the dust particle's electrostatic potential more positive (see for example Chow et al. 1995). Furthermore, the suprathermal electrons present in the interplanetary medium can make the dust particle potential multivalued, i.e. there are two stable equilibrium potentials of opposite signs for a given dust particle in a given environment, so that an infinitesimal variation in properties can yield jumps in potential and similar dust particles can have charges of opposite signs (Meyer-Vernet 1982).

Secondly, dust particles are generally not spherical, so that the equilibrium charge is greater than the value (12). In practice, for non-spherical dust particles of volume $V$, (12) still holds with the effective radius $R_{d}=(3 V / 4 \pi)^{1 / 3}$, provided that the length-to-diameter ratio is smaller than about ten, but the charge may be much greater for long rods and fluffy dust particles (Auer 2007).

Thirdly, even plasma current collection can be more complex than the simple expression given by eq. (5) in environments such as the lower ionosphere because of the presence of different ionic species including negative ions (e.g. Rapp et al. 2005).

For very small dust particles, further processes come into play. In the interplanetary medium, the potential is limited by field emission if the surface electric field $\sim \Phi / R_{d}$ (where $R_{d}$ is the dust particle's radius or the smallest value of the curvature radius for a non-spherical dust particle) is greater than the value enabling the dust particle's electrons or ions to tunnel towards the surface. For positive potentials, only ion emission can take place, so that the critical field strength $\sim 3 \cdot 10^{10}$ volts $/ \mathrm{m}$ is reached only for dust particles smaller than a fraction of nm. However in that case, the electrostatic stress $\sim \varepsilon_{0}\left(\Phi / R_{d}\right)^{2}$ generally exceeds the maximum tensile strength against fracture (itself generally smaller than $\sim 10^{9} \mathrm{~N} / \mathrm{m}^{2}$ - the value for tektites or polycrystalline bulk solids) (see for example Hill and Mendis 1981), so these dust particles are generally disrupted before ion emission can take place. The sub-nm dust is also possibly ionized due to electron collision or can be charged by electron attachment (Watanabe 2006). Finally, one sees from (13) and (14) that dust particles smaller than a few tens $\mathrm{nm}$ and a few $\mathrm{nm}$ in respectively the ionosphere and the interplanetary medium may have a charge smaller than unity, so that charge fluctuations are comparable to the charge itself. In that case, the charging process becomes statistical (Watanabe 2006; see also Rapp et al. 2005; Draine and Sutin 1987). Note that photo emission and photo-detachment were recently discussed for meteoric smoke composed of metal oxides (e.g., Rapp 2009). 
5.2 Laboratory experiments and related numerical models

While several laboratory experiments have been carried out to study the parameters of grain ensembles within a plasma, experiments at single grains are still a challenge. We start by describing the experimental set ups to manipulate the single particles (5.2.1) and after discussing the scattered measurements of different charging processes (5.2.2) report about the detailed studies of the secondary electron emission (SEE) process (5.2.3), of the dust exposed to ion beams (5.2.4) and of dust sputtering (5.2.5).

\subsubsection{Experimental set ups}

Most measurements at single cosmic dust analogs are carried out based on the quadrupole trap technique, which was developed in the 1950s for ion spectroscopy (Paul and Steinwedel 1956). The Dusty Plasma Laboratory at Marshall Space Flight Center, Huntsville, Al, USA uses a quadrupole trap at pressures $\approx 10^{-3}-10^{-2} \mathrm{~Pa}$. In this device the dust surface charge is derived from the electrostatic force in the trap indicated by the compensating dc potential applied to the balance electrodes in order to levitate the particle (Spann et al. 2001, Abbas et al. 2001, 2004). The group studied charging of Apollo 17 dust grains exposed by lowenergy electron beams (Abbas et al. 2008). This device is also used for radiation pressure measurements and for studying grain alignment and grain rotation(see Abbas et al. 2003), which are beyond the scope of our discussion.

Since the laboratory measurements bear the difficulty of separating the effects of the remaining atmospheric species in the device from the measured effect, the Space Physics Laboratory at Charles University, Prague, has developed a 3D quadrupole trap that operates under ultra-high vacuum conditions $\left(\approx 10^{-7} \mathrm{~Pa}\right.$ or better). In this device the grain charge is derived from the recorded oscillation frequency of the grain that is (due to the specific shape of the electrodes) proportional to its charge-to-mass ratio (Cermak et al., 1994). Special techniques are also applied to measure the grain mass, diameter, and capacitance (Cermak et al. 1995, Zilavy et al. 1998, Pavlu et al. 2004). Also Grimm et al. (2006) studied the charging of dust particles in a high vacuum quadrupole trap, in this case the grains were exposed to soft $\mathrm{x}$ rays. The quadrupole trap measurements are typically carried out at grains of several $100 \mathrm{~nm}$ size. The challenges in studying smaller grains lie in handling the small particles, measuring their size and reducing effects due to the remaining pressure in the device.

Experimenters in the Dusty Plasma Group at University of Colorado at Boulder followed a different concept in which particles are dropped through the plasma volume and the dust surface charge is derived from the voltage pulses measured at a Faraday cup at the bottom of the volume (see Walch et al. 1994, 1995). This method detects an ensemble of grains and results can only be interpreted only statistically.

\subsubsection{Measurements of different charging processes}

This section briefly reviews investigations of interactions that are not discussed in detail in the following. Several laboratory experiments have been carried out to study specific issues of the grain charging within a plasma. Barkan et al. (1994) studied the difference in charging of micron-sized grains in a dense dust cloud to the charge on an isolated grain. Walch et al. (1994, 1995) measured the charge on small grains of non-conducting materials in a plasma containing both thermal and suprathermal electrons. Sickafoose et al. (2001) studied the photoemission and triboelectric charging of single dust grains levitating upon a 
metal surface. Triboelectric charging which is the transfer of charge via mechanical contact, is an important charging process for planetary regolith analogs, as are secondary electron emission and photo electron emission. Triboelectric charging is also suggested as one of the mechanisms that generate secondary charges in the rocket- borne dust detectors (see section 6.1). Abbas et al. (2006) presented laboratory measurements of the photoelectric yields of grains of silica, olivine, and graphite of $0.1-5 \mu \mathrm{m}$ radii illuminated with a UV radiation at $120-160 \mathrm{~nm}$ wavelengths. Large grains (tens of micrometers) had an order-of-magnitude higher yields than that reported for planar samples. Submicron grains with sizes several wavelengths of the incident photons had yields close to or below the bulk value and so far there is no agreement with theoretical results. Small size effects are also important for the secondary emission of electrons (SEE), which gains importance for small grains.

\subsubsection{Secondary electron emission experiments (SEE)}

SEE effects are usually underestimated but SEE is important whenever the energetic (above $50 \mathrm{eV}$ ) electrons are present. Theoretical descriptions of SEE still follow Sternglass (1957) who established a formula hat describes the yield of the secondary electron emission, i.e. the number of ejected electrons per infalling electron, as a function of the energy of the primary electron beam. The description bases on measurements at large planar samples. This yield rises with the beam energy to a maximum (at $0.3-2 \mathrm{keV}$ ) and then drops down to zero. The height of the maximum depends on the material. It is about unity for metals and reaches several tens for some insulators. Electrons leaving the grain consist of two different populations: true secondary electrons and backscattered primary electrons. The energy distribution of true secondary electrons is limited to about $50 \mathrm{eV}$, whereas scattered electrons can reach energies comparable to that of the primary electron beam. Suszcynsky and Borovsky (1992) modified the Sternglass theory based on measurements at electron beam energy 2-30 keV and at different materials, in particular insulators and ices.

The secondary emission of electrons is very sensitive to the surface curvature (Draine and Salpeter 1979). The yield increases with incident angle because the main interaction region is closer to the surface. Thus particles with highly curved surfaces and small particles with size comparable to the beam have enhanced yields (Richterova et al. 2007). For very small grain size, the beam electrons are not captured inside the grain and the surface potential can reach rather large values (e.g., Pavlu et al. 2008, Beranek et al. 2010a).

The increase of grain charges for small sizes is a common feature that is observed for many different materials (Svestka et al. 1993, Richterova et al. 2006a, Richterova et al. 2007, Beranek et al. 2009). This was expected from theoretical studies who show that SEE yield increases with decreasing size and becomes very large for grains with size of the order of the primary electron penetration depth. The measured surface potentials, however, exceeds that results from SEE. Richterova et al. (2004) simulated numerically secondary electron emission from spherical dust grains and found that backscattered electrons have considerable influence on the equilibrium surface potential, especially for high primary energies. The authors have improved the model including more complex principles of the electron-solid interaction and using an advanced Monte Carlo technique (Richterova et al. 2006a). The results can be briefly summarized as follows: (1) the scattering of primary electrons inside the grain is critical to understanding the secondary electron emission, (2) the grain charge is determined not only by the SEE yield, but equally important is the energy distribution of secondary electrons, (3) the increase of the surface potential with decreasing grain size is predominantly caused by the increasing number of backscattered primary electrons, not by true secondary electrons. The model was successfully verified by laboratory experiments on 


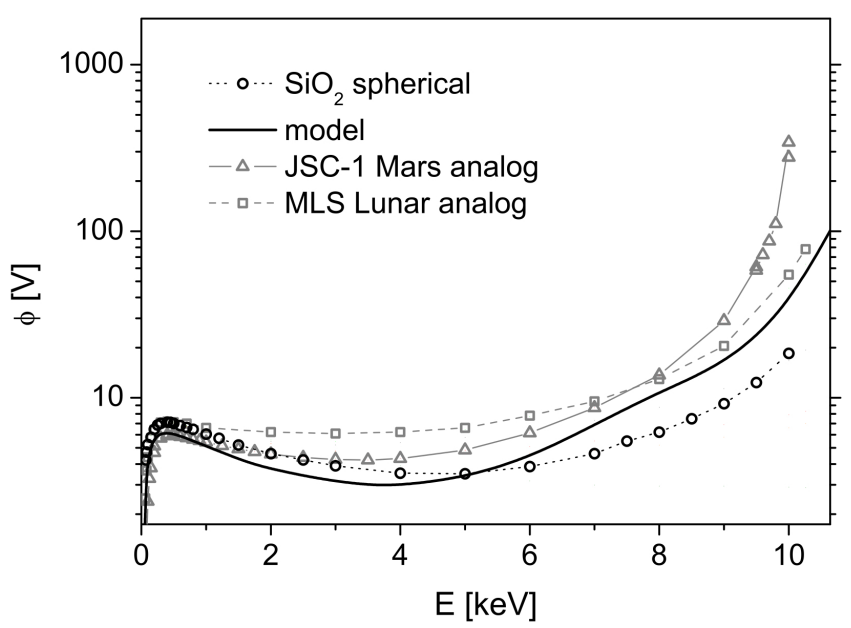

Fig. 10 Grain surface potentials, $\phi$ as a function of the primary electron beam energy for the JSC Mars-1 and MLS Lunar analogs together with the results calculated according the Richterova et al. (2010b) model for glass spheres with diameter of $0.6 \mu \mathrm{m}$ and corresponding mass $0.25 \cdot 10^{-15} \mathrm{~kg}$. The masses of measured grains are: $\mathrm{SiO}_{2}-1.3 \cdot 10^{-15} \mathrm{~kg}$, JSC-1 Mars $-1.5 \cdot 10^{-15} \mathrm{~kg}$, and MLS Lunar $-1.8 \cdot 10^{-15} \mathrm{~kg}$.

spherical grains from different materials (Richterova et al. 2006a, 2006b, 2007, Beranek et al. 2009, Pavlu et al., 2009).

The measured surface potentials of Martian and Lunar analog grains are shown together with the results of the Richterova et al. (2006a) model in Fig. 10. The potential profiles roughly follow the profile of the secondary emission yield at low (below $1.5 \mathrm{keV}$ ) primary energies for all investigated grains. The masses and compositions of the grains are similar and the differences observed in higher energies can be attributed to the shape effect. To show it, we used the experimental measurements for $\mathrm{SiO}_{2}$ spheres (Richterova et al. 2007) because this component is a principal constituent of both simulants. A comparison shows that the grain from the Lunar simulant behaves approximately as the $\mathrm{SiO}_{2}$ sphere of a smaller (approx. by a factor of 2) size. It suggests that a better approximation of the Lunar sample shape would be an ellipsoid of revolution with the ratio of axes of about $1 / 2$ and even smaller ratio would be appropriate for the Martian sample. A similar shape effect was found in Pavlu et al. (2008) where the charging of grain clusters was widely discussed. The positive grain potential can reach several hundred of volts and it is limited by the threshold of the ion field emission.

Motivated by the Cassini observation of ice dust grains with a salt admixture in the Saturnian E-ring (Postberg et al. 2009), Richterova et al. (2010a) used the last modification of the secondary emission model (Richterova et al. 2010b) to study the secondary emission yield and surface potential of icy dust grains as a function of the grain size and the salt admixture (see Fig. 11). They find that the potential of the salty grain differs only very slightly from that calculated for the grain from pure water ice for any reasonable salt admixture and suggest that the presence of the salt changes only the scattering of the primary electrons inside the grain but does not affect the emission of the true secondary electrons. Hence, the parameters for the pure ice can be used for estimation of the charging and the presence of the salt can be neglected. Experimental data on ice dust grains are still missing, though.

The discussed measurements were carried out at grains with small surface charge. The grain potential, however, influences SEE. Even more, a large negative electric field at the 


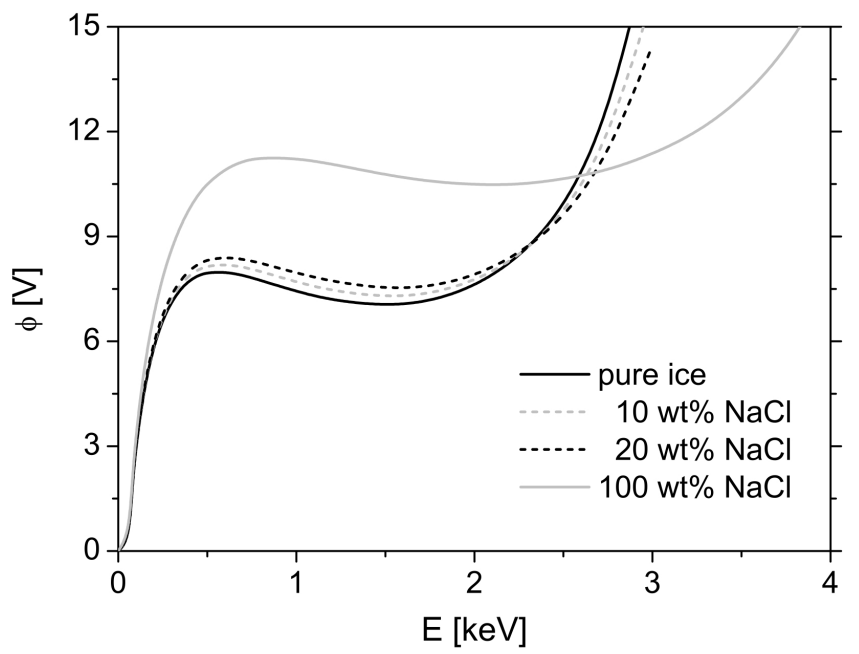

Fig. 11 The surface potentials, $\phi$ as a function of the primary electron beam energy for ice with different admixtures of $\mathrm{NaCl}$. The admixture is given in percents of weight.

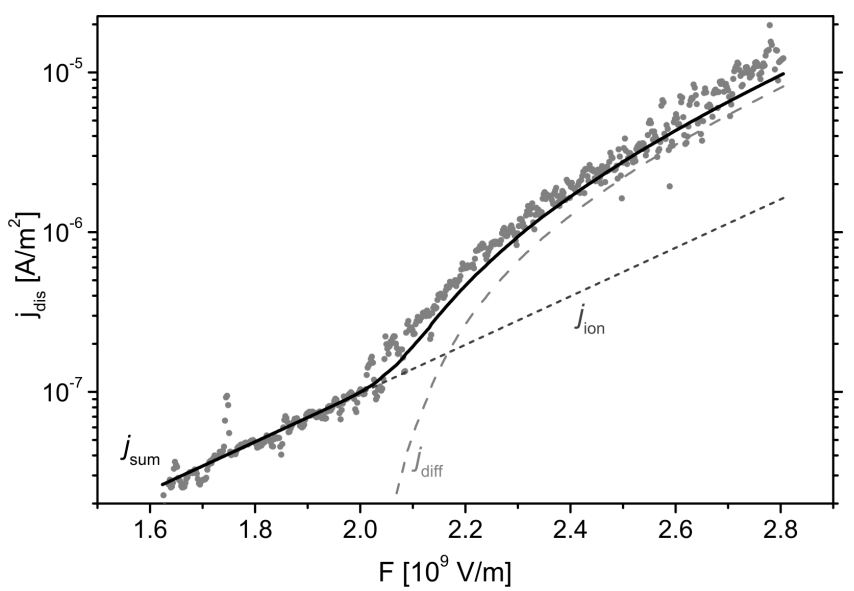

Fig. 12 Comparison of the measured discharging of the carbon grain with the simple model of FIE. The discharging current, $j_{d i s}$ is composed of two terms: $j_{i o n}$ and diffusion current, $j_{\text {dif }}$ and their sum is denoted as $j_{\text {sum }}$. (Adopted from Jerab et al. (2010b). Reprinted with permission (c)2010 IEEE.)

grain surface modifies electronic structure of the surface states and through increase of the escape probability of excited electrons change the SEE (Beranek et al. 2009).

\subsubsection{Ion beam experiments}

Another class of laboratory experiments was devoted to interaction of the dust with energetic ions. It is generally expected that ion beams would charge the dust grain to a potential equal to the beam energy but Cermak et al. (1995) have shown that a high grain potential can lead to spontaneous discharging, which is possibly attributed to field evaporation of bulk material. Sternovsky et al. (2001) found a threshold electric field of the order of $10^{8} \mathrm{~V} / \mathrm{m}$ 


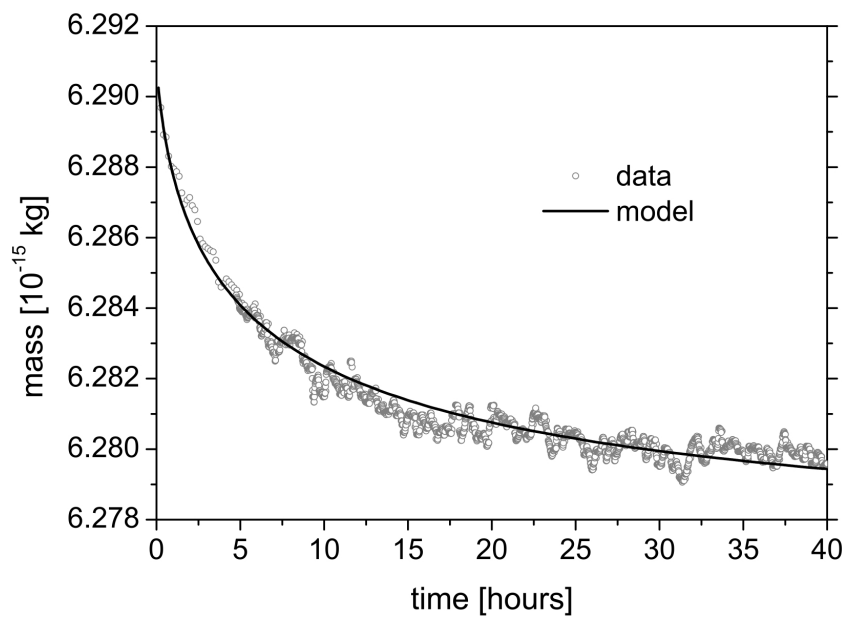

Fig. 13 The change of the grain mass in time. The parameters of the model $\left(D=5 \cdot 10^{-16} \mathrm{~cm}^{2} / s\right.$, treatment: $8 \mathrm{~h}$, incident current: 32400 particles per second). The horizontal axis indicates the time after treatment switch-off. (Adopted from Beranek et al. (2010b). Reprinted with permission (C)2010 IEEE.)

for the field evaporation current. The authors concluded that this process can lead to ion emission from submicron grains exposed to solar UV radiation.

New systematic experiments show that the threshold electric field as well as the discharging current depend on the charging history (Velyhan et al. 2004, Pavlu et al. 2006). The experiments used the melamine-formaldehyde spheres as dust grain samples and the structure of this material can be easily destroyed by the ion bombardment. For this reason, Jerab et al. (2007) repeated the charging experiment using gold samples. They found that the discharging current increases with the dose of ions applied to the grain. This effect was attributed to an implantation of the beam ions into the grain surface and their backward diffusion and consecutive field ionization of desorbed atoms. This conclusion was confirmed by further measurements that used carbon dust grains and Ar ions (Jerab et al. 2010). The authors developed a simple model of the grain discharging that takes into account the field ionization of the residual atmosphere in the vacuum vessel and diffusion and field ionization of the implanted ions. A good agreement between the model and experiment is demonstrated in Fig. 12 where the discharging current is plotted as a function of the surface electric field (Note that actually both quantities change in time). The discharging current is supposed to be a sum of two components: the field ionization current, $j_{i o n}$ and diffusion current, $j_{d i f}$.

Beranek et al. (2010b) performed a quantitative investigation of ion implantation. They used the combination of light carbon grains and heavy argon ions and measured directly the change in grain mass after prolonged ion bombardment. The results plotted in Fig. 13 show time constants of this process of the order of 10 hours at room temperature. Since the diffusion coefficients depend on the temperature exponentially, they concluded that the dust grains keep the implanted ions over long periods of time.

\subsubsection{Sputtering experiments}

Sputtering experiments are difficult and time consuming because the ion beam intensity should be kept low; otherwise the ion drag would blow the grain from the trap. Pavlu et al. (2007) bombarded a gold grain with Ar ions for 250 hours and this bombardment led to a 
decrease of the grain mass by about one half. A model developed for an interpretation of the results revealed two factors that distinguish the sputtering yield of small grains from that measured on planar surfaces under the same conditions. First of them is the shape effect that increases sputtering yield by a factor of $\approx 2$. The second factor is the grain charge resulting in the intense electric field at the grain surface. The electric field enhanced the sputtering yield by another factor of $\approx 2.5$. Based on these results, Pavlu et al. (2008) computed the life-time of grains under typical conditions in the interplanetary space and found a value of $\approx$ $5.10^{2}$ years as a typical value for sputtering of a micron sized grain. The authors considered an average elemental composition of the solar wind and they have shown that heavy ions contribute to the overall sputtering yield by about one half due to their large efficiency. However, we should note that the real sputtering yield would be even higher because the influence of the implanted ions on the grain mass was neglected in the experiment and it can be significant. The sputtering rates derived from the laboratory measurement are higher than those discussed in section (4.1) and this is due to the sputtering from the heavy solar wind species. Another effect that was found and that was not taken into account in calculations is the ion drag effect. Energetic ions are often present as directional beams in the planetary magnetospheres or interplanetary space. The ion drag orients the grains along the longest axis into the beam direction and thus a large portion of the grain surface is bombarded by the ions with a small incident angle. Depending on the shape of a particular grain, this effect can bring a further increase of the sputtering rate by a factor of 2-5. The resulting dust lifetimes are still of the order of the Poyinting - Robertson lifetime, but the results shows that sputtering is more important than suggested by the theoretical considerations.

\section{Dust in-situ measurements}

Most of the instruments designed for in-situ detection of dust particles rely on either measurements of the dust primary surface charge or on measurements of the charge generated by the dust impact on a target. Knowledge of the dust charging and of the dust impact processes is therefore essential, and the limitations of available theory and experiment poses currently a limit to the data evaluation. Moreover, the Langmuir probe type dust measurements are in principle limited. Likewise the detection of dust with plasma wave measurements requires understanding the impact generated cloudlets and their evolution in the solar wind plasma. Optical detection methods are rarely applied and limited due to the small signal of scattered light in the case of the smaller dust, as well as by the time resolution of the light detection and by the requirements for deep space probes.

We discuss the dedicated dust instruments on sounding rockets in the ionosphere (6.1) and on deep space missions (6.2) and finally the dust detection with plasma experiments (6.3).

\subsection{Dust instruments on sounding rockets}

Dust can be observed in situ from sounding rockets in the ionosphere and this can in principle be made by mass spectrometry, like for the ions. Arnold et al. (1982) discuss the connection to a meteoric source in the context of measuring heavy ions $(\mathrm{m}>100 \mathrm{amu})$ from sounding rocket. A possible detection of meteoric smoke particles from sounding rocket was reported by Schulte and Arnold (1992) based on quadrupole mass spectrometry measurements. They detected a layer of negative ions with masses up to $400 \mathrm{amu}$ and above and 
suggested that the ions "are best explained as being the negatively charged fraction of the so-called meteor smoke particles". Since then, dust measurements were made from rockets at altitudes roughly 70 to $100 \mathrm{~km}$. The first measurements of dust particles with a dedicated instrument from sounding rocket was reported by Havnes et al. (1996). This instrument (as do later measurements e.g. Horanyi et al. 2000) detects the current due to the surface charge of impacting particles. The instrument contains two entrance grids that are biased to positive, respectively negative potential relative to the charge-collecting Faraday cup. The grid-induced field deflects charged species below a certain mass threshold to enter the detector and prevents the detection of electrons and light ions. Similarly Gelinas et al (1998) subtract the dust current from the larger background current with an oscillating electric field that alternately deflects the dust to two different anodes. The deflection only applies to a specific mass interval of particles. A similar concept with an applied alternating voltage was used to dected positively and negatively charged dust (Lynch et al. 2005). Also other deflection mechanisms were used. Some experiments combine the charge measurements with Langmuir probe measurements in order to better discriminate the ion and electron signals from the dust signals (Mitchell et al. 2001, Goldberg et al. 2001). Improved instruments applying primary charge detection are equipped with an UV flash lamp to enhance the dust charge by photoionisation and hence lower the threshold of detected dust particles (Rapp et al. 2003, 2010). Measurements with UV photometers of the solar light scattered at dust provide information on dust downward to $40-50 \mathrm{~nm}$ size and indicate the existence of smaller dust (Gumbel et al. 2001), but optical detection is limited by the light scattering efficiency of the dust particles at the wavelength of observation.

The dust instruments that measure primary charge detect, similarly to Langmuir probes the currents in a plasma, the combined current of ions, electrons and dust charge. They do not allow single particle detection and the dust masses are derived assuming a specific surface charge of the dust; hence the derived numbers are based on assumptions concerning the charging process. The data are possibly contaminated by secondary charges: The dust impact velocities range from several $100 \mathrm{~m} / \mathrm{s}$ to $1 \mathrm{~km} / \mathrm{s}$ and the dust impacts may generate free charges. This was already pointed out in the context of the first measurements (Havnes 1996) and Havnes and Næsheim (2007) re-evaluted the conditions of the measurements. For a different rocket experiment Barjatya and Swenson (2006) find that the data are influenced by triboelectric charging of the dust at the payload surface and they question in the reliability of Langmuir probe - type dust measurements in general.

Measurements from sounding rocket are moreover principally limited by the aerodynamics. Horanyi et al.(1999) find in a study for a specific experimental configuration that nano dust is deflected from entering the detector and that measurements of ice particles are further influenced by sublimation and condensation. Gumbel et al.(2001) point out that this may reduce the detection efficiency of the nano dust with sizes $<10 \mathrm{~nm}$ by 10 to $100 \%$ depending on the individual detection geometry and atmospheric conditions and that further interactions within the instrument may influence the signal. They suggest combined dust flux and aerodynamic studies to attack this problem. Considering the aerodynamics of the meteoric smoke is further complicated by the fact that the payload moves from continuum flow to free molecular flow conditions (Hedin et al. 2007a).

Collecting dust samples would allow to prove the origin of the dust and this is the purpose of a rocket campaign project (Gumbel et al. 2005). A sampling device was designed to collect dust from a sounding rocket under minimized aerodynamical perturbations (Hedin et al. 2007b), but this failed so far for various technical reasons. 
6.2 Dust instruments on deep space spacecraft

Dust in the interplanetary medium is predominantly observed with impact ionization detectors (for the technical and functional principles see Auer 2001, for a review of current and future dust in-situ measurements see Grün et al. 2005). The particles are destroyed during the impact and the produced electrons and ions that originate from the dust and from the detector target are measured. For each dust impact a sequence of different charge signals is measured within the instrument and allows to distinguish dust signals from those of other particles that possibly reach the instrument. The charges are typically measured at the entrance grids, the target and the sidewalls. Charge amplitudes provide information about the ionized mass fraction and the time sequence of the signals about the impact speed, if combined with pre-flight laboratory calibration measurements. Combination of the impact speed with the detector geometry and spacecraft velocity and orientation at the time of the event allow estimating the dust velocities.

Detailed numerical studies of the impact ionization process were performed (Hornung et al. 2000 and references therein). Laboratory measurements of impact generated charges are carried out during instrument calibration. Numerical and experimental results agree with the following rough picture: At impact velocities $1-50 \mathrm{~km} / \mathrm{s}$, typical in the interplanetary medium, collisions lead to fragmentation as well as to the partial ionization of dust and target surface materials. At impact velocities beyond $50 \mathrm{~km} / \mathrm{s}$ the entire particle is destroyed and the degree of ionization of the produced vapor increases with impact velocity. For large impact velocities and small particles the detectors provide reliable information about dust mass and in some cases relative velocities. Major limitation of the measurements lie in the complexity of the impact ionization process and in the range of dust impact velocities, which do not extend above $100 \mathrm{~km} / \mathrm{s}$ in laboratory measurements. Moreover, the dust masses, materials and structures that can be prepared for laboratory calibration measurements are limited (see also section 6.3).

For recent measurements in the interplanetary medium(see Krüger et al. 2006 and references there) the mass range of particles for which the mass could be derived is $10^{-19}$ $10^{-10} \mathrm{~kg}$ and the determined velocity has a factor of 2 uncertainty and the mass a factor of 10 uncertainty. Note, that a significant fraction of registered events is caused by impacts of fast and small dust particles outside of the range of instrument calibration.

The element composition of the dust can be inferred in principle from impact ionization experiments measuring the time of flight of the produced ions. The detected species originate from complex ionization, expansion and recombination processes and this complicates the analysis. So far a full quantitative analysis of dust composition in space has not been achieved. The mass spectra generated by dust impacts were measured and analyzed during the space missions to comet Halley and also in the interplanetary medium with the Cosmic Dust Analyser (CDA) experiment onboard the Cassini spacecraft (see Hillier et al. 2007; Postberg et al. 2008).

In-situ measurement of dust surface charges in the interplanetary medium were firstly achieved also with CDA (Kempf et al. 2004). The dust passes a system of entrance grids while entering CDA and thereby induces a charge signal. In the case of 6 reported impact events the dust charge was sufficiently large to generate a clear feature of induced charge at the grid system and this allowed to derive the surface charge. The measured surface charges range between $1.3-5.4 \cdot 10^{-15} \mathrm{C}$. The mass of the particles is uncertain. The authors suggested their results agree with theoretical models of dust surface charging: assuming a surface potential of $+5 \mathrm{~V}$ for the dust, they find that the detected dust particles have a mass 
larger than $10^{-13} \mathrm{~kg}$ and that the number of detected particles agrees with model predictions in this mass range.

\subsection{Dust detection with plasma experiments}

Dust detection by plasma instruments is based on measuring the voltage induced on an electric antenna when a dust particle impacts a spacecraft at high speed and then vaporises and ionizes, as well as a part of the targets material. This produces a plasma cloud whose electric charges are detected. The released charge which is much larger than that normally carried by the dust particle induces a large voltage on the electric antenna (Fig. 14).

Such a detection was made serendipitously for the first time on the spacecraft Voyager 1 in Saturn's E ring (Aubier et al. 1983; Gurnett et al. 1983), and was later used in various environments of the heliosphere (see Meyer-Vernet 2001 and references therein).

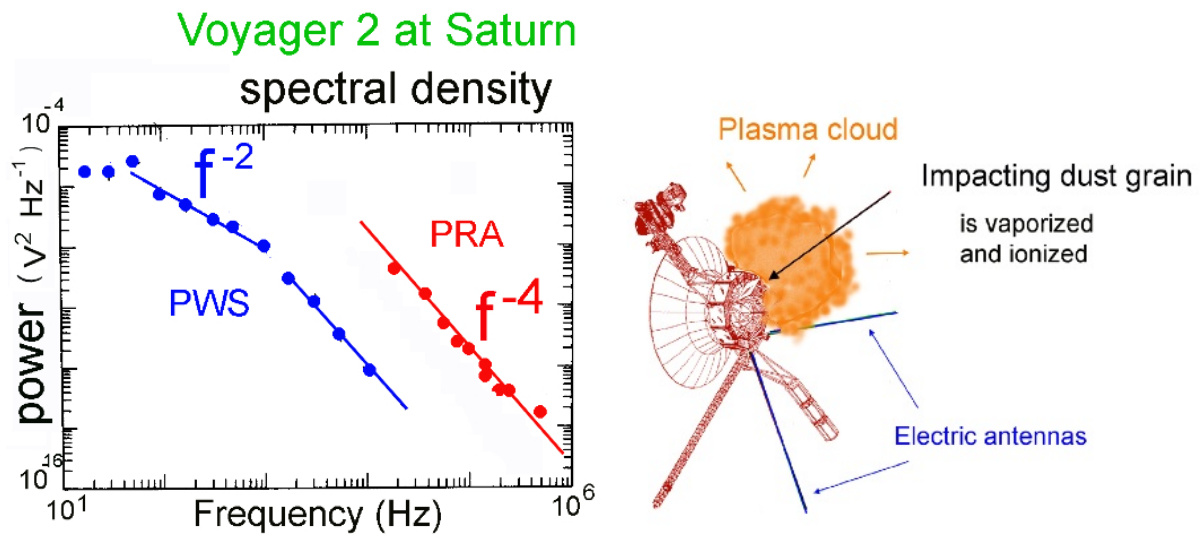

Fig. 14 A dust particle impacting a spacecraft at a high speed vaporises and ionizes; this produces an expanding plasma cloud whose charge is partially recollected, inducing a transient voltage on the electric antennas. The voltage spectral density measured on Voyager 2 by the radio (denoted as PRA) and plasma wave instruments (denoted as PWS). The spectrum varies as $f^{-4}$ at high frequencies and $f^{-2}$ at lower frequencies. Because of the asymmetrical antenna configuration, the amplitude is much greater when the antennas are in monopole mode (the case of the Voyager PRA instrument).

This process is basically the same as for classical impact ionization detectors (Section 6.2) with, however, two important differences. Firstly, since the wave instruments on present space probes were generally not designed to measure dust, they were not calibrated for that purpose, so that the dust measurement is generally not accurate - a problem which may be solved on future space probes. However, this technique has the advantage of having a much larger effective area than conventional dust detectors, since it is of the order of magnitude of the cross-section of the spacecraft itself, which generally amounts to several squared meters. The charge released upon impact increases fast with the impact speed (see Section 4.1). This produces voltage pulses on the antenna, since charges are recollected by the spacecraft or antennas and an electric field is produced. The voltage $V$ measured as a function of $Q$ depends on the antenna/spacecraft configuration and on the impact site; it rises with a time scale $\tau_{r}$, which can be evaluated from simple physical arguments (Meyer-Vernet 1986; Oberc 1994, 1996), and subsequently decays with a longer time scale $\tau_{d}$ determined 
by the system time constant. Measuring these impulses with a wave-form receiver reveals the mass and impact rate of the particles. At frequencies $f \gg 1 / 2 \pi \tau_{r}$, the Fourier transform $|V(\omega)|$ is determined by the discontinuity of the derivative in the rising part, so that it is given by

$$
|V(\omega)|=V_{\max } /\left(\tau_{r} \omega^{2}\right)
$$

Hence, the total voltage power spectral density $V_{\omega}^{2}$ at high frequencies is (Meyer-Vernet 1996)

$$
V_{\omega}^{2}=2\left\langle N V_{\max }^{2} / \tau_{r}^{2}\right\rangle / \omega^{4}
$$

where $N$ is the impact rate, the brackets stand for an average over the dust mass distribution, and the factor of two stems from the fact that the spectral density is defined for positive frequencies only. This yields a power spectrum varying as $1 / f^{4}$ at high frequencies, whose level can be used to deduce the dust mass distribution. At frequencies intermediate between the inverse decay time and the inverse rise time, the signal behaves as a step function, producing a $1 / f^{2}$ power spectrum, which is not adequate for detecting dust because it may be confused with genuine plasma signals having a similar spectral index (Meyer-Vernet and Perche 1989).

The corresponding spectral density is shown on Fig. 14, as recorded with the radio (denoted as PRA; Aubier et al. 1983; Meyer-Vernet et al. 1998) and plasma wave (denoted as PWS; Gurnett et al. 1983; Tsintikidis et al. 1994) instruments on Voyager 2. When the antenna is operated as a dipole, the receiver records the difference of potential between the two antenna arms. Hence, except when the antenna is short and/or the arms are not symmetrically located with respect to the spacecraft, it responds weakly to dust impacts on the spacecraft and this response depends on the asymmetries of the system. This is illustrated on Fig. 14, where the signal of the plasma wave instrument (PWS, operating as a dipole) is much smaller than the one recorded by the radio instrument (PRA, operating as a monopole, i.e. measuring the difference of potential between an antenna arm and the spacecraft).

This technique has been recently applied to the measurement of nanoparticles in the solar wind near Jupiter with the wave instrument (RPWS) on the spacecraft Cassini (MeyerVernet et al. 2009a) and at 1 AU with the WAVES instrument on STEREO (Meyer-Vernet et al. 2009b)

Two other techniques have been proposed to detect dust with plasma instruments, which do not require the dust particles to impact the spacecraft. They are based on the transient voltage that the charge carried by the particle induces on an electric antenna when it passes closer to it than the ambient Debye length. The dust particles can be detected individually by a wave-form analysis (Meuris et al. 1996). The main advantage of this latter technique is that it has an extremely large equivalent area for dust detection - roughly the product of the antenna antenna length by the Debye length, which amounts typically to $100 \mathrm{~m}^{2}$ in the interplanetary medium. Since, however, the voltage induced is small and might be confused with genuine plasma wave effects, this method requires a very sensitive receiver and an elaborate analysis. When the dust concentration is sufficiently high that numerous flybys are detected simultaneously, the wave receiver records a voltage power spectrum which is a superposition of the spectra produced by individual particle flybys near the antenna (MeyerVernet 2001). The principle is similar to the electron measurements performed routinely from plasma thermal noise spectroscopy in space (Meyer-Vernet and Perche 1989), but the calculation is easier because the dust particles generally move so slowly that the plasma temporal dispersion plays a negligible role. 


\section{Summary and discussion:}

In spite of meteors well visible in the night sky, at present we do not know very well the amount and composition of meteoroid species that are fed into the atmosphere. The observation of radar meteors in general is an important tool to better quantify the influx of meteoroid mass into the Earth atmosphere and its spatial and temporal variations. We can roughly estimate the overall element composition of the entering meteoroids from cosmic abundance considerations and laboratory analyses of collected samples of dust and meteorites provide information about the solids that survive the entry process. Meteor spectra provide further information, but do not reveal the full meteoroid composition.

The perspectives of future observations of dusty plasma effects are diverse. Theory is still insufficient to explain the observed phenomena and knowledge from dust experiments and from laboratory data is limited. Dust charging and dust impact processes play an important role in the dust plasma interactions, but also for the understanding of dust in-situ instruments. In both cases there is a gap between the theory and the laboratory experiments and furthermore the laboratory experiments do not fully reproduce space conditions.

Dusty plasma effects comprise (i) the alteration of the dust as a result of the ambient plasma (charging, erosion, condensation and growth), (ii) the generation of neutrals, electrons or ions from dust, (iii) the change in energy or charge state of plasma species by interaction with the dust, (iv) the dust motion or change of motion as a result of the ambient plasma, and (v) collective behavior caused by the presence of dust. The latter is often denoted as dusty plasma in the narrow sense of the expression. We summarize our review according to these listed dusty plasma effects ( $\mathrm{i}-\mathrm{v}$ ) and will point out options for future space experiments and theoretical and experimental studies.

Referring to (i), the alteration of dust in the Earth's ionosphere in the case of meteoroid ablation is inferred from the observed meteor phenomenon. The surface charge of dust is (in a few cases) detected with in-situ dust measurements (both in interplanetary space and Earth's ionosphere). At present, however, the measurements are subject to a large uncertainty. We are not aware of direct measurements of dust erosion, dust condensation and dust growth in natural cosmic environments (aside from experiments under microgravity conditions, which are beyond the scope of this review). A major limitation is the paucity of laboratory data on the interactions of solids (such as secondary electron emission yields, sputtering yields) and limited knowledge of the small particle effects that may influence the interaction. Direct measurements of the dust surface charge will be most likely possible with improved space dust in-situ measurements.

The generation of neutrals, electrons or ions from dust (ii) is possibly detected in the interplanetary medium via the solar wind pick up ions. The vapor production by mutual dust collisions is a major mechanism. The generation of neutrals, electrons or ions from dust leads to the different meteor phenomena in the Earth's ionosphere. Experimental techniques have recently improved enough to carry out detailed spectroscopic meteor observations, which are a promising tool to shed some light on the nature of the generated species. Such studies may also lead to information about the composition and, perhaps, the shapes of meteoroids. We point out that a consistent physical model to describe meteor spectra is still missing, though.

The change in energy or charge state of plasma species by interaction with the dust (iii) is suggested (aside from the release of dust species) in order to explain pick up ions and also neutral solar wind, but so far the observed species could not be attributed to a dust related process in a quantitative way. Also in the case of high altitude meteors such processes play a role. Future experimental studies that may lead to some progress are the observational 
studies of high altitude meteors, as well as the simultaneous in- situ detection of dust and plasma components in the interplanetary medium.

Referring to (iv), the dust submicrometer-sized dust is significantly influenced by radiation pressure and gravitational forces. Fast nano dust particles accelerated by solar wind interactions have also been detected. Though the forces acting on dust in a plasma are well known, the uncertainty in the parameters that determine these forces hampers a quantitative discussion.

Referring to (v), many theoretical studies consider plasma collective effects in the Earth's ionosphere and enhanced dust densities in meteor trails potentially may lead to waves and instabilities. The development of quantitative models is limited by the lack of experimental data concerning the detection of dust in the nm size range. As a result of the small dust number densities compared to the ambient solar wind plasma densities plasma collective effects are not likely to occur in the interplanetary medium on a large scale, though they may occur locally, as for instance in the vicinity of comets. The collective effects in meteor trails may in the future be possibly detected by radar observations.

In summary we identify the following issues for future research on dust plasma interactions: (a) ion, atom, and molecule interactions on dust surfaces in the limit of low energies (sputtering, recombination, molecular reactions); (b) the charging mechanisms of nano-dust; (c) the dynamics of nano-dust including the influence of charge fluctuations; (d) the impact ionization process of nano-dust; (e) the sublimation of nano-dust. These research issues require theoretical considerations combined with laboratory measurements. The study of high altitude meteors may further reveal dust plasma interactions as well as dusty plasma collective effects, though as a result of the number of contributing parameters (like dust size, composition, charging processes) these seem to be more complex than predicted by current theory.

Progress in space measurements is expected from the in-situ detection of nano particles and of dust surface charges in the ionosphere and interplanetary medium, the detection of plasma waves related to charged dust or dust impacts and the detection of ion species generated by dust impact in the Earth's ionosphere and in the interplanetary medium.

Acknowledgements This article was prepared within an International Team at the International Space Science Institute, ISSI, Bern, Switzerland. Parts of this work were supported by the German Aerospace Center DLR (Deutsches Zentrum für Luft- und Raumfahrt) under project (RD-RX / 50 QP 0403) and by the Japanese Ministry of Education, Science, Sports and Culture, Grant-in-Aid for Scientific Research -C 19540475, 2008. This collaboration was also partly supported by the Swedish Foundation for International Cooperation in Research and Higher Education, STINT (I.M. and E.M.) and by the US DOE Grant No. DE-FG02-04ER54804 and NSF Grant no. PHY-0903808 NSF and ATM-0907941 (MR) and by the Open Research Center at Kindai University. We acknowledge helpful discussions with Markus Rapp in the initial stage of this project and we thank Dennis Höning for his support in preparing the manuscript.

\section{References}

1. M. Abbas, P. Craven, J. Spann, E. West, J. Pratico, D. Tankosic, and C. Venturini, Phys. Scr. T98, 99-103 (2001).

2. M. Abbas, P. Craven, J. Spann, D. Tankosic, A. LeClair, D. Gallagher, E. West, J. Weingartner, W. Witherow, and A. Tielens, Astrophys. J. 614(2), 781-795 (2004).

3. M. M. Abbas, D. Tankosic, P. D. Craven, J. F. Spann, A. LeClair, E. A. West, J. C. Weingartner, A. G. G. M. Tielens, J. A. Nuth, R. P. Camata, and P. A. Gerakines, Astrophys. J. 645 (1), 324-336 (2006).

4. M. M. Abbas, D. Tankosic, J. F. Spann, M. J. Dube, and J. A Gaskin, Space Technology and Applications International Forum-STAIF 2008, AIP, CP969, 2008.

5. C.W. Allen, Astrophysical Quantities, 3rd Edn. (London, Athlone, 1976) 
6. Arnold et al. (1982)

7. M.G. Aubier, N. Meyer-Vernet, B.M. Pedersen, Geophys. Res. Lett. 10, 5 (1983)

8. S. Auer, in Astron. Astrophys. Library, ed. By E. Grün, B.A.S. Gustafson, S. Dermott, H. Fechtig (Springer, Berlin and New York, 2001) pp. 385-444

9. S. Auer, in Workshop on Dust in Planetary Systems. September 26-30 2005, Kauai, Hawaii. ed. By H. Krüger and A. Graps (ESA SP, 2007) p. 231-233

10. P.B. Babadzhanov, Astron. Astrophys. 384, 317-321 (2002)

11. A. Barjatya and C.M. Swenson, J. Geophys. Res. 111, A10302, doi:10.1029/2006JA011806 (2006)

12. P.M. Banks, J. Geophys. Res. 19, 4341 (1971)

13. A. Barkan, N. D’Angelo, and R. Merlino, Phys. Rev. Lett. 73, 3093-3096 (1994).

14. R. Behrisch and W. Eckstein, in Topics Appl. Physics 110, ed. By R. Behrisch, W. Eckstein (Springer, Berlin Heidelberg, 2007)

15. L.R. Bellot Rubio, M.J. Martninez Gonzalez, L.R. Herrera, J. Licandro, D.M. Delgado, P.R. Gil, M. Serra-Ricart, Astron. Astrophys. 389, 680-691(2002)

16. M. Beranek, I. Richterova, Z. Nemecek, J. Pavlu, and J. Safrankova, Eur. Phys. J. D, 54 (2), 299-304 (2009).

17. M. Beranek, M. Vysinka, J. Pavlu, I. Richterova, Z. Nemecek, J. Safrankova, IEEE Trans. Plasma Sci. 38 (4), 886-891 (2010a).

18. M. Beranek, J. Pavlu, J. Vaverka, I. Richterova, J. Safrankova, and Z. Nemecek, J. Geophys. Res., in press, 2010b.

19. P.A. Bernhardt, G. Ganguli, M.C. Kelley, W.E. Swartz, J. Geophys. Res. 100, 23811 (1995)

20. D. Bilitza and B.Reinisch, J. Adv. Space Res. 42, 599-609 (2007) doi:10.1016/j.asr.2007.07.048

21. J. Borovička, Astron. Astrophys. 279, 627-645 (1993)

22. J. Borovička, in Meteorids 1998, ed. By W.J. Baggaley and V. Porubcan (Astron. Inst., Slovak Acad. Sci., 1999) pp. 355-363

23. Borovicka, J., Roya. J.: Astron. Soc. Canada 100 (2006) 194198.

24. M. Bzowski and M. Krlikowska, in: (B. Fleck et al. eds.) Proceedings of the Solar Wind 11 / SOHO 16 Conference, ESA SP-592) 397-400 (2005)

25. U. Brändström, The Auroral Large Imaging System - Design, Operation, and Scientific Results, IRF Sci. Rep. 279, Swedish Institute of Space Physics, Kiruna (2003)

26. V.A. Bronshten, Physics of Meteoric Phenomena, (D. Reidel. Publ.Co., Dordrecht, 1983), p. 356

27. N. Brosch, L.S. Schijvarg, M. Podolak, M.R. Rosenkrantz, in Proceedings of the Meteoroids 2001 Conference, 6-10 August 2001, Kiruna, Sweden, ed. By B. Warmbein, (ESA Publications Division, Noordwijk, 2001), pp. 165-173

28. N. Brosch, D. Polishook, R. Helled, S. Schijvarg, M. Rosenkrantz, Atmos. Chem. Phys. 4, 1063-1069 (2004)

29. P.G Brown, M.D. Campbell, R.L Hawkes, C. Treijsmeijer, J. Jones, Planet. Space Sci. 50, 45-55 (2002)

30. M. D. Campbell-Brown, Earth, Moon, and Planets, 95, 521-531 (2004)

31. M.D. Campbell-Brown and D. Koschny, Astron. Astrophys. 418, 751-758 (2004)

32. M.D. Campbell-Brown and S. Close, Monthly Notices of the Royal Astronomical Society 382, 1309$1316(2007)$

33. M.D Campbell, P.G. Brown, A.G. LeBlanc, R.L. Hawkes, J. Jones, S.P. Worden, R.R. Correll, Meteoritics \& Planetary Science 35, 1259-1267 (2000)

34. J.D. Carpenter, T.J. Stevenson, G.W. Fraser, J.S. Lapington, D. Brandt, J. Geophys. Res. 110 (2005)

35. Z. Ceplecha, J. Borovička, W. G. Elford, D. O. Revelle, R. L. Hawkes, V. Porubcan, M. Simek, Space Sci. Rev. 84, 327-471 (1998)

36. I. Cermak, E. Grün, and J. Svestka, Adv. Sp. Res. 15(10), 59-64 (1995)

37. E. Chapin and E. Kudeki, Geophys. Res. Lett. 21, 2433 (1994)

38. J.Y.N. Cho and J. Röttger, J. Geophys. Res. 102, 2001-2010 (1997)

39. V.W. Chow and M. Rosenberg, Phys. Plasmas 3, 1202 (1996)

40. V. Chow, D. Mendis, and M. Rosenberg, J. Geophys. Res. 98, 19065 (1993).

41. V. Chow, D. Mendis, and M. Rosenberg, IEEE Trans. Plasma Sci. 22, 179 (1994).

42. V.W. Chow, et al., Astrophys. Space Sci. 223, 144 (1995)

43. S. Close, S.M. Hunt, M.J. Minardi, F.M. McKeen, Radio Science 35, 1233-1240 (2000)

bibitemCollier2003 M.R. Collier, T.E. Moore, K. Ogilvie, D.J. Chornay, J. Keller, S. Fuselier, J. Quinn, P. Wurz, M. Wüest, K.C. Hsieh, AIP Conference Proceedings, 679, 790-793 (2003)

44. S.G. Coulson, Monthly Notices of the Royal Astronomical Society 332, 741-744 (2002)

45. S.G. Coulson, N.C. Wickramasinghe, Monthly Notices of the Royal Astronomical Society 343, 1123$1130(2003)$

46. C.L. Croskey, J.D. Mitchell, M. Friedrich, K.M. Torkar, U.P. Hoppe, R.A. Goldberg, Geophys. Res. Lett. $28,1427-1430(2001)$ 
47. A. Czechowski and I. Mann, Astrophys. J. 714, 89-99 (2010)

48. P. Czechowsky, R. Ruester, G. Schmidt, Geophys. Res. Lett. 6, 459-462 (1979)

49. D.J. Cziczo, D.S. Thomson, D.M. Murphy, Science 291, 1772-1775 (2001)

50. N. D’Angelo, Planet. Space Sci. 38, 1143 (1990)

51. N. D’Angelo, Planet. Space Sci. 51, 393 (2003)

52. N. D’Angelo, Phys. Lett. A 336, 204 (2005)

53. J.S. Dohnanyi, in: Cosmic Dust (J. A. M. McDonnell, Ed.), pp. 527-606. Wiley, Chichester (1978)

54. B. Draine and E. Salpeter, Astrophys. J. 231(1), 77-94 (1979).

55. B.T. Draine and B. Sutin, Astrophys. J. 320, 803 (1987)

56. J. Duprat, C. Engrand, M. Maurette, G. Kurat, M. Gounelle, C. Hammer, Adv. Space Res. 39 (4), 605611(2007).

E. Dubinin, M. Fraenz, J. Woch, J. Winnigham, R. Frahm, R. Lundin, and S. Barabash, Planet. Space. Sci. $56(6), 846-851(2008)$

57. L.P. Dyrud, M.M. Oppenheim, A.F. vom Endt, Geophys. Res. Lett. 29, 2012 (2002)

58. L. Dyrud, D. Wilson, S. Boerve et al. Adv. Space Res. 42, 136-142 (2008)

59. H.J. Fahr, H.W. Ripken, G. Lay, Astron. Astrophys. 102, 359 (1981)

60. V.E. Fortov, A.V. Ivlev, S.A. Khrapak, A.G. Khrapak, G.E. Morfill, Phys. Reports 421, 1 (2005)

61. Y. Fujiwara, M. Ueda, Y. Shiba, M. Sugimoto, M. Kinoshita, C. Shimoda, Geophys. Res. Lett. 25, 285288 (1998)

62. T. Fukuzawa, S. Hashim, Y. Matsuoka, et al., J. Appl. Phys. 86, 3543 (1999)

63. P. Gabrielli, C. Barbante, J. Plane et al., Nature 432, 1011-1014 (2004)

64. L.J. Gelinas, K.A. Lynch, M.C. Kelley, S. Collins, S. Baker, Q. Zhou, J.S. Friedman, Geophys. Res. Lett. 25, 4047-4050 (1998)

65. L.J. Gelinas, K.A. Lynch, M.C. Kelley, R.L. Collins, M. Widholm, E. MacDonald, J. Ulwick, P. Mace, J. Geophys. Res. 110 (2005)

66. G. Gloeckler, L.A. Fisk and J. Geiss, in: (M. Maksimovic, K. Issautier, N. Meyer-Vernet, M. Moncuquet,

F. Pantelliniet Eds.) AIP CP 1216, 514-517 (2010)

67. C.K. Goertz, Rev. Geophys. 27, 271 (1989)

68. R.A. Goldberg, R.F. Pfaff, R.H. Holzworth, et al. Geophys. Res. Letters 28 no. 8, 1407-1410 (2000)

69. R.J.L. Grard, J. Geophys. Res. 78, 2885 (1973)

70. E. Grün, H.A. Zook, H. Fechtig, R.H. Giese, Icarus 62, 244 (1985)

71. E. Grün, R. Srama, H. Krüger, et al., Icarus 174, 1 (2005)

72. J. Gumbel, Journ. Geophys. Res. 106 no A6, 10553-10563 (2001)

73. J. Gumbel, K.W.T. Waldemarsson, F. Giovane, et al., Proc. 17th ESA Symposium on European Rocket and Balloon Programmes and Related Research (ESA SP-590), 139-144 (2005)

74. D.A. Gurnett, E. Grün, D. Gallagher, et al., Icarus 53, 236 (1983)

75. O. Havnes, Astron. Astrophys. 193, 309 (1988)

76. O. Havnes and F. Sigernes, Journal of Atmospheric and Solar-Terrestrial Physics 67, 659-664 (2005)

77. O. Havnes and L.I. Næsheim, Ann. Geophys., 25, 623637 (2007)

78. O. Havnes, T. Aslaksen, A. Brattli, Phys. Scripta T89 133 (2001)

79. O. Havnes, J. Trøim, T. Blix, W. Mortensen, L.I. Næsheim, E. Thrane, T. Tønnesen, J. Geophys. Res. 101, 10839-10848 (1996)

80. R.L. Hawkes and J. Jones, Monthly Notices of the Royal Astronomical Society 173, 339-356 (1975)

81. R.L. Hawkes, in Meteors in the Earth's Atmosphere, ed. By E. Murad, E. and I. P. Williams (Cambridge University Press, 2002), pp. 97-122

82. R.L. Hawkes, P.G. Brown, N.R. Kaiser, A.J. Faloon, K.A. Hill, L.A. Rogers, EMP 95, 587-593 (2004)

83. A.E. Hedin, J. Geophys. Res. 96, 1159, (1991)

84. J. Hedin, J. Gumbel, and M. Rapp, Atmos. Chem. and Physics 7, 3701-3711 (2007)

85. J. Hedin, J. Gumbel, T. Waldemarsson, and F. Giovane, Adv. Space Res. 40, 818-824 (2007)

86. J.R. Hill and D.A. Mendis, Canad. J. Phys. 59, 897 (1981)

87. K.A. Hill, L.A. Rogers, R.L. Hawkes, Earth, Moon, and Planets 95, 403-412 (2005)

88. J.K. Hillier, S.F. Green, N. McBride et al., Icarus 190, 643-654 (2007)

89. R.H. Holzworth, R.F. Pfaff, R.A. Goldberg, et al., Geophys. Res. Lett. 28, 1435 (2001)

90. M. Horanyi and D.A. Mendis, Astrophys. J. 294, 357-368 (1985)

91. M.S. Horanyi, B. Robertson, J. Smiley, G. Gumbel, G. Witt, B. Walch, Geophys. Res. Lett. 27, 38253828 (2000)

92. M.S. Horanyi, Annu. Rev. Astron. Astrophys. 34, 383418 (1996)

93. K. Hornung, Y.G Malama, K.S Kestenboim, Astrophys. Space Sci. 274, 355-363 (2000)

94. D.W. Hughes, in Meteors and Cosmic Dust, ed. By J. A. M. McDonnell (Wiley-Interscience, Hoboken,

N. J, 1978), pp. 123-186

95. D.M. Hunten, R.P. Turco, O.B. Toon, J. Atmos. Sci. 37, 1342-1357 (1980) 
96. M. Jerab, I. Richterova, J. Pavlu, J. Safrankova, and Z. Nemecek, IEEE Trans. Plasma Sci. 35 (2), 292296 (2007).

97. M. Jerab, J. Vaverka, M. Vysinka, Z. Nemecek, J. Safrankova, IEEE Trans. Plasma Sci. 38 (4), 798-802 (2010).

98. J. Jones and A.R. Webster, Planet. Space Sci. 39, 873-878 (1991)

99. A.P. Jones, A. G. G. M. Tielens, and D. J. Hollenbach, D. J., Astrophys. J. 469, $740-764$ (1996).

100. G.H. Jones, A. Balogh, D. J. McComas, and R. J. MacDowall, Icarus 166, 297-310 (2003).

101. A. G. G. M. Tielens, C.F. McKee, C.G, Seab, and D. J. Hollenbach, D. J. Astrophys. J., 431, 321340(1994).

102. O. Kalashnikova, M. Horanyi, G.E. Thomas, et al., Geophys. Res. Lett. 27, 3293 (2000)

103. T.J. Kane and C.S. Gardner, Science 259, 1297-1300 (1993)

104. M.C. Kelley, The Earth's Ionosphere: Plasma Physics and Electrodynamics, (Academic Press, San Diego, 1989)

105. M.C. Kelley, C. Alcala, J.Y.N. Cho, Journal of Atmospheric and Solar-Terrestrial Physics 60, 359-369 (1998)

106. M.C. Kelley, Radio Sci. 39, RS2015 (2004)

107. S. Kempf, R. Srama, N. Altobelli, et al., Icarus 171, 317 (2004)

108. J. Kero, Swedish Institute of Space Physics, PhD theses, Kiruna, Sweden (2008)

109. J. Kero, C. Szasz, G. Wannberg, A. Pellinen-Wannberg, A. Westman, Geophys. Res. Lett. 35 (2008a) doi:10.1029/2008GL033402

110. J. Kero, C. Szasz, A. Pellinen-Wannberg, G. Wannberg, A. Westman, D. D. Meisel, Geophys. Res. Lett. 35 (2008b) doi:10.1029/2007GL0327333

111. J. Kero, C. Szasz, A. Pellinen-Wannberg, G. Wannberg, A. Westman, "3D position determination of small coherent meteor targets with EISCAT UHF," accepted for publication in Annales Geophysicae, 2008c 112. H. Kimura, I. Mann, E.K. Jessberger, Astrophys. J. 582, 846-858 (2003)

113. P. Koten, J. Borovika, P. Spurný, H. Betlem, S. Evans, Astron. Astrophys. 428, 683-690 (2004)

114. P. Koten, P. Spurný, J. Borovičcka, S. Evans, A. Elliott, H. Betlem, R. Štork, K. Jobse, Meteoritics \& Planetary Science $41,1305-1320$ (2006)

115. A.R. Krauss and D.M. Grün, J. Nuclear Materials 85-86, 1179-1183 (1979)

116. H. Krüger, N. Altobelli, B. Anweiler, S.F. Dermott, V. Dikarev, A. Graps, E. Grün, B.A. Gustafson, D.P. Hamilton, M.S. Hanner, M. Horanyi, J. Kissel, M. Landgraf, B.A. Lindblad, G. Linkert, I. Mann, J.A.M. McDonnell, G. Morfill, C. Polanskey, G. Schwehm, R. Srama, H.A. Zook, Planet. Space Sci. 54, 932-956 (2006)

117. A. Kull, E. Kopp, C. Granier, G. Brasseaur, J. Geophys. Res. 102, 9705-9716 (1997)

118. J.G. Laframboise and L. W.Parker, Phys. Fluids, 16, 629 (1973)

119. Ch, Leinert, S. Bowyer, L.K. Haikala, et al. Astron. Astrophys. Suppl. 127, 1-99 (1998)

120. J. Lemaire, Astrophys. J. 360, 288 (1990)

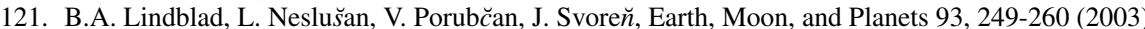

122. S.G. Love and D.E. Brownlee, Science 262, 550-553 (1993)

123. K.A. Lynch et al., J. Geophys. Res. 110 (2005) A03302 doi: 10.1029/2004JA010502

124. I. Mann, in Landolt Boernstein Handbook Series, LB VI/4B: Chap. 4.3.2., ed. By J. Trümper (Springer, Heidelberg, 2009)

125. I. Mann and A. Czechowski, Astrophys. J. 621, L73-L76 (2005)

126. I. Mann, E. Murad, A. Czechowski, System, Planet. Space Sci. 55, 1000-1009 (2007)

127. I. Mann, H. Kimura, D. A. Biesecker, B. T. Tsurutani, E. Grün, B. McKibben, J. C. Liou, R. M. MacQueen, T. Mukai, L. Guhathakurta, P. Lamy, Space Sci. Rev. 110, 269 (2004)

128. I. Mann, A. Czechowski, and N. Meyer-Vernet, N. in: (M. Maksimovic, K. Issautier, N. Meyer-Vernet, M. Moncuquet, F. Pantelliniet Eds.) AIP CP 1216, 491-496 (2010a)

129. I. Mann, A. Czechowski, and N. Meyer-Vernet, A. Zaslavsky, H. Lamy H., Plasma Phys. Control. Fusion 52, in print (2010b)

130. G.G. Mateshvili, Y.D. Mateshvili, N.Y. Mateshvili, Sol. Syst. Res. 31, 483-488 (1997)

131. N. Mateshvili, G. Mateshvili, I. Mateshvili, L. Gheondjian, O. Avsajanishvili, Meteoritics \& Planetary Science 34, 969-973 (1999)

132. J.D. Mathews, D. Janches, D.D. Meisel, Q.-H. Zhou, Geophys. Res. Lett. 28 , 1929-1932 (2001)

133. N. McBride and J.A.M. McDonnell, Planet. Space Sci. 47, 1005 (1999)

134. D.W.R. McKinley, Meteor Science and Engineering, (McGraw-Hill, New York, 1961)

135. W.J. McNeil, S.T. Lai, E. Murad, J. Geophys. Res. 103, 10899 - 10911 (1998)

136. W.J. McNeil, E. Murad, J.M.C. Plane, in Meteors in the Earth's Atmosphere, ed. By E. Murad and I. P. Williams (Cambridge University Press, 2002), pp. 265-287

137. L. Megner, D.E. Siskind, M. Rapp, J. Gumbel, J. Geophys. Res. 113 D03202, doi:10.1029/2007JD009054 (2008) 
138. L. Megner, M. Rapp, J. Gumbel, Atmos. Chem. Phys. 6, 4415 (2006)

139. D.A. Mendis and M. Rosenberg, Annu. Rev. Astron. Astrophys. 32, 419 (1994)

140. P. Meuris, N. Meyer-Vernet, J. F. Lemaire, J. Geophys. Res. 101, 24471-24478 (1996)

141. R.A. Mewaldt, C.M.S. Cohen, G.M. Mason, et al. Space Sci. Rev. 130, 207-219 (2007)

142. N. Meyer-Vernet, Astron. Astrophys. 105, 98 (1982)

143. N. Meyer-Vernet, Adv. Space Res. 5, 37 (1986)

144. N. Meyer-Vernet, in Proc. 7th Spacecraft Charging Technology Conf. ed. By R. A. Harris, ESA SP-476 (2001), pp. 635-640

145. N. Meyer-Vernet, Basics of the Solar Wind, Cambridge University Press (2007)

146. N. Meyer-Vernet and C. Perche, J. Geophys. Res. 94, 2405 (1989)

147. N. Meyer-Vernet, A. Lecacheux, B.M. Pedersen, Icarus 123, 113 (1996)

148. N. Meyer-Vernet, A. Lecacheux, B.M. Pedersen, Icarus 132, 311-320 (1998)

149. N. Meyer-Vernet, A. Lecacheux, M.L. Kaiser, D.A. Gurnett, Geophys. Res. Letters 36, 3103 (2009a)

150. N. Meyer-Vernet, M. Maksimovic, A. Czechowski, I. Mann, I. Zouganelis, K. Goetz, M.L. Kaiser, J.-L. Bougeret, S.D. Bale, Dust detection by the wave instrument on Stereo: nanoparticles picked-up by the solar wind?, Solar Phys. 256, 463-474 (2009b)

151. T. Minato, M. Köhler, H. Kimura, I. Mann, T. Yamamoto, Astron. Astrophys. 424, L13-L16 (2004)

152. T. Minato, M. Köhler, H. Kimura, I. Mann, T. Yamamoto, Astron. Astrophys. 452, 701 (2006)

153. N.Y. Misconi, J. Geophys. Res. 98, 18 951-18 961 (1993)

154. J.D. Mitchell, D.J. Walter, C.L. Croskey, R.A. Goldberg, ESA SP-370, 95-100 (1995)

155. J.D. Mitchell, C.L. Croskey, R.A. Goldberg, Geophys. Res. Letters 28 no 8, 1423-1426 (2000)

156. G.E. Morfill and E. Grün, Planet. Space Sci. 27, 1269-1292 (1979)

157. T. Mukai, Astron. Astrophys. 99, 1 (1981)

158. T. Mukai and G. Schwehm, Astron. Astrophys. 95, 373-382 (1981)

159. T. Mukai and T. Yamamoto, Astron. Astrophys. 107, 97-100 (1982)

160. S. Mukai, I. Sano, M. Yasumoto, Adv. Space Res. 32, 2181-2190 (2003)

161. T.G. Northrop, Adiabatic Motion of Charged Particles, Wiley, NewYork (1963)

162. P. Oberc, Icarus 111,211 ( 1994)

163. P. Oberc, Adv. Space Res 17, 110 (1996)

164. E.J. Öpik, Physics of Meteor Flight in the Atmosphere, (Interscience Publishers, New York, 1958), p. 174

165. M.M. Oppenheim, L.P. Dyrud, A.F. vom Endt, J. Geophys. Res. 108, 1064 (2003)

166. E.N. Parker, Astrophys. J. 128, 664 (1958)

167. W. Paul and H. Steinwedel, Apparatus for separating charged particles of different specific charges, Patent 944,900, German Patent, 1956.

168. J. Pavlu, A. Velyhan, I. Richterova, Z. Nemecek, J. Safrankova, I. Cermak, and P. Zilavy, IEEE Trans. Plasma Sci. 32(2), 704-708 (2004).

169. J. Pavlu, A. Velyhan, I. Richterova, J. Safrankova, Z. Nemecek, J. Wild, and M. Jerab, Vacuum 80 (6), 542547 (2006).

170. J. Pavlu, I. Richterova, Z. Nemecek, J. Safrankova, J. Wild, IEEE Trans. Plasma Sci., 35 (2), 297-302, (2007).

171. J. Pavlu, I. Richterova, Z. Nemecek, J. Safrankova, and I. Cermak, Faraday Discuss. 137, 139-155 (2008).

172. J. Pavlu, J. Safrankova, Z. Nemecek, and I. Richterova, Contrib. Plasma Phys. 49 (3), 169186 (2009).

173. A. Pellinen-Wannberg, Atmos. Chem. Phys. 4, 649-655 (2004)

174. A. Pellinen-Wannberg and G. Wannberg, J. Geophys. Res. 99, 11379-11390 (1994)

175. A. Pellinen-Wannberg ans G. Wannberg, Journal of Atmospheric and Terrestrial Physics 58, 495-506 (1996)

176. A. Pellinen-Wannberg, G. Wannberg, J.Kero, C. Szasz and A. Westman, URSI Radio Scienc Bulletin 324 (2008)

177. A. Pellinen-Wannberg, E. Murad, B. Gustavsson, U. Brändström, C.-F. Enell, C. Roth, I.P. Williams, Å. Steen, Geophys. Res. Lett. 31 (2004) doi: 10.1029/2003GL018785

178. A. Pellinen-Wannberg, E. Murad, N. Brosch, I. Haggstrom, T. Khayrov, in (J.A. Fernandez, D. Lazzaro, D. Prialnik R. Schulz, eds.), Icy Bodies of the Solar System Proceedings IAU Symposium No. 263, International Astronomical Union, doi:10.1017/S1743921310001869 (2010)

179. B. Peucker-Ehrenbrink, Geochim. Cosmochim. Acta 60, 3187-3196 (1996)

180. J.M.C. Plane, Annales Geophysicae 18, 807-814 (2000)

181. J.M.C. Plane, in Meteors in the Earth's Atmosphere, ed. By E. Murad, E. and I.P. Williams (Cambridge University Press, 2002), pp. 289-309

182. J.M.C. Plane, C.S. Gardner, J. Yu, et al. J. Geophys. Res. 104, 3773-3788 (1999)

183. Y. V. Platov, S. A Chernouss, M. J. Kosch, J. Spacecraft Rockets 41, 667 (2004) 
184. O.P. Popova, Earth, Moon, and Planets 95, 303-319 (2004)

185. O.P. Popova, A.S. Strelkov, S.N. Sidneva, Adv. Space Res. 39, 357 (2007)

186. F. Postberg, S. Kempf, J.K. Hillier, R. Srama, S.F. Green, N. McBride, E. Grün, Icarus 193, 438-454 (2008)

187. F. Postberg, S. Kempf, J. Schmidt, N. Brilliantov, A. Beinsen, B. Abel, U. Buck, and R. Srama, Nature 459 (7250), 1098-1101 (2009).

188. B.R. Ragot and S.W. Kahler, Astrophys. J. 594, 1049 - 1059 (2003)

189. N.N. Rao, P.K. Shukla, M.Y. Yu, Planet. Space Sci. 38, 543 (1990)

190. M. Rapp, Ann. Geophys. Vol. 27, 2417-2422 (2009)

191. M. Rapp and F.J. Lübken, Atmos. Chem. Physics 4, 2601 (2004)

192. M. Rapp, F.J. Lubken, T.A. Blix, Adv. Space Res. 31, 2033 (2003)

193. M. Rapp, I. Strelnikova, B. Strelnikov, et al., Joun. Geophys, Res. 115, D00I16 (2010)

194. M. Rapp, J. Hedin, I. Strelnikova, et al., Geophys. Res. Lett. 32, L23821 (2005)

195. I. Richterova, Z. Nemecek, J. Safrankova, and J. Pavlu, IEEE Trans. Plasma Sci. 32(2), 617-622 (2004).

196. I. Richterova, J. Pavlu, Z. Nemecek, and J. Safrankova, Phys. Rev. B 74(23), 235430 (2006a).

197. I. Richterova, J. Pavlu, Z. Nemecek, J. Safrankova, and P. Zilavy, Adv. Sp. Res. 38(11), 2551-2557 (2006b).

198. I. Richterova, Z. Nemecek, J. Safrankova, J. Pavlu, and M. Beranek, IEEE Trans. Plasma Sci. 35(2), 286-291 (2007).

199. I. Richterova, M. Beranek, J. Pavlu, Z. Nemecek, and J. Safrankova, Icarus, in press, 2010a.

200. I. Richterova, M. Beranek, J. Pavlu, Z. Nemecek, and J. Safrankova, Phys. Rev. B 81(7), 075406 (2010b).

201. F.J.M. Rietmeijer in: (Murad E and Williams I P) Meteors in the Earth's atmosphere (Cambridge: Cambridge University Press) p 215 (2002)

202. R. Roble, in Energetics of the mesosphere and thermosphere in AGU Monograph, The Upper Mesosphere and Lower Thermoshphere: A review of experiment and theory. 87, ed By R. M. Johnson, T. M. Killeen (American Geophysical Union, Washington DC, 1995), pp. 1-20

203. L.A. Rogers, K.A. Hill, R.L. Hawkes, Planet. Space Sci. 53, 1341-1354 (2005)

204. M. Rosenberg, Planet. Space Sci. 41, 229 (1993)

205. M. Rosenberg, Planet. Space Sci. 56, 1190-1193 (2008)

206. M. Rosenberg and V.W. Chow, Planet. Space Sci. 46, 103 (1998)

207. M. Rosenberg and P.K. Shukla, J. Geophys. Res. 105, 23135 (2000)

208. M. Rosenberg and P.K. Shukla, Planet. Space Sci. 50, 261 (2002)

209. M. Rosenberg and G. Sorasio, J. Spacecr. Rockets 43, 245 (2006)

210. M. Rosenberg and R.L. Merlino, Planet. Space Sci. 55, 1464 (2007)

211. M. Rosenberg, M. Salimullah, R. Bharuthram, Planet. Space Sci. 47, 1517 (1999)

212. J. Rosinski and R.H. Snow, J. Meteorol. 18, 736 (1961)

213. C. T. Russell, L. K. Jian, H. R. Lai, T.L. Zhang, A. Wennmacher, J. G. Luhmann, in: (M. Maksimovic,

K. Issautier, N. Meyer-Vernet, M. Moncuquet, F. Pantelliniet Eds.) AIP CP 1216, 522-525 (2010).

214. W.A. Scales and G. Ganguli, New J. Phys. 6, 12 (2004)

215. R.W. Schunk and A.F. Nagy Ionospheres: Physics, Plasma Physics, and Chemistry (Cambridge University Press, 2000)

216. N.A. Schwadron, J. Geiss, L.A Fisk, G. Gloeckler, T.H. Zurbuchen, R. von Steiger, J. Geophys. Res. $105,7465(2000)$

217. Schulte and Arnold (1992)

218. H. Seiler, J. Appl. Phys. 54, R1 (1983).

219. P.K. Shukla and V.P. Silin, Phys. Scripta 45, 508 (1992)

220. P.K. Shukla and A.A. Mamun, Introduction to Dusty Plasma Physics, (IOP Publ., Bristol, 2002)

221. A. Sickafoose, J. Colwell, M. Hornyi, and S. Robertson, J. Geophys. Res. 106(A5), 8343 (2001).

222. J. Spann, M. Abbas, C. Venturini, and R. Comfort, Phys. Scr. T89, 149-153 (2001).

223. P. Spurný, J. Borovička, P. Koten, Earth, Moon, and Planets 95, 569-578 (2004)

224. O.C. St.Cyr, S.P. Plunkett, D.J. Michels, S.E. Paswaters, M.J. Koomen, G.M. Simnett, B.J. Thompson,

J.B. Gurman, R. Schwenn, D.F. Webb, E. Hildner, P.L. Lamy, J. Geophys. Res. 105,18169-18186 (2000)

225. E. Sternglass, Scientific Paper 6-94410-2-P9, Westinghouse Research Laboratories, Pittsburgh 35 (1957).

226. Z. Sternovsky, Z. Nemecek, J. Safrankova, and A. Velyhan, IEEE Trans. Plasma Sci. 29 (2), 292-297 (2001).

227. D. M. Suszcynsky and J. E. Borovsky, Phys. Rev. A 45, 6424, (1992).

228. D. M. Suszcynsky, J. E. Borovsky, and C. K. Goertz, J. Geophys., Res., Solid Earth 97, 2611 (1992).

229. J. Svestka, I. Cermak, and E. Grün, Adv. Sp. Res. 13(10), 199-202 (1993).

230. C. Szasz, J. Kero, A. Pellinen-Wannberg, et al., Earth, Moon, and Planets 95 (1-4), 101-107 (2004) 
231. C. Szasz, J. Kero, A. Pellinen-Wannberg, D.D. Meisel, G. Wannberg, A. Westman, Earth, Moon, and Planets doi:10.1007/s11038-007-9206-y (2007)

232. C. Szasz, J. Kero, D.D. Meisel, A. Pellinen-Wannberg, G. Wannberg, A. Westman, Monthly Notices of the Royal Astronomical Society 388, 15-25 (2008)

233. S. Taylor, J. H. Lever, R. P. Harvey, Nature 392, 899-903 (1998)

234. A. G. G. M. Tielens, C.F. McKee, C.G, Seab, and D. J. Hollenbach, D. J. Astrophys. J., 431, 321340(1994).

235. D. Tsintikidis, D. Gurnett, L.J. Granroth, et al., J. Geophys. Res. 99, 2261 (1994)

236. V.M. Vasyliunas and G.L. Siscoe, J. Geophys. Res. 81, 1247-1252 (1976)

237. A. Velyhan, P. Zilavy, J. Pavlu, J. Safrankova, and Z. Nemecek, Vakuum 76 (4), 447-455 (2004)

238. F. Verheest, Waves in dusty space plasmas, Kluwer Academic Publishers, Dordrecht (2000)

239. D. Vinkovic, Adv.Space Res. 39, 574-582 (2007)

240. T. Vondrak, J.M.C. Plane, S. Broadley, D. Janches. Atmos. Chem. Phys. 8, 7015-7031 (2008)

241. B. Walch, M. Horanyi, and S. Robertson, IEEE Trans. Plasma Sci. 22(2), 97-102 (1994)

242. B. Walch, M. Hornyi, and S. Robertson, Phys. Rev. Lett. 75(5), 838-841 (1995)

243. G. Wannberg, A. Pellinen-Wannberg, A. Westman, Radio Science 31, 497-518 (1996)

244. Y. Watanabe, J. Phys. D: Appl. Phys. 39, R329R361 (2006)

245. A. Wehry and I. Mann, Astron. Astrophys. 341, 296-303 (1999)

246. E.C. Whipple, Rep. Prog. Phys. 44, 1197 (1981)

247. D.M. Wood, Phys. Rev. Lett. 46, 749 (1981)

248. A.M. Zadorozhny, A.A. Tyutin, G. Witt, et al., Geophys. Res. Lett. 20, 2299 (1993)

249. Q.H. Zhou and M.C. Kelley, J. Atm. Solar Terr. Phys. 59, 739 (1997)

250. P. Zilavy, Z. Sternovsky, I. Cermak, Z. Nemecek, and J. Safrankova, Vacuum 50(1-2), 139-142 (1998). 